\title{
Article
}

\section{Aspects of quantum energy and stress in inhomogeneous unbounded dielectric continua}

Goto, Shin-itiro, Tucker, Robin W. and Walton, Timothy

Available at http://clok.uclan.ac.uk/39739/

Goto, Shin-itiro, Tucker, Robin W. and Walton, Timothy ORCID: 0000-00015103-4591 (2018) Aspects of quantum energy and stress in inhomogeneous unbounded dielectric continua. Reviews in Mathematical Physics, 31 (1). ISSN 0129-055X

It is advisable to refer to the publisher's version if you intend to cite from the work. http://dx.doi.org/10.1142/S0129055X19500028

For more information about UCLan's research in this area go to http://www.uclan.ac.uk/researchgroups/ and search for <name of research Group>.

For information about Research generally at UCLan please go to http://www.uclan.ac.uk/research/

All outputs in CLoK are protected by Intellectual Property Rights law, including Copyright law. Copyright, IPR and Moral Rights for the works on this site are retained by the individual authors and/or other copyright owners. Terms and conditions for use of this material are defined in the policies page.

\section{CLoK}

Central Lancashire online Knowledge www.clok.uclan.ac.uk

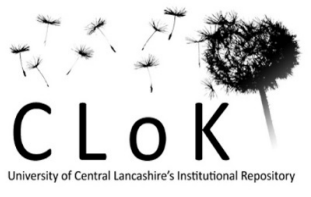




\title{
Aspects of quantum energy and stress in inhomogeneous unbounded dielectric continua
}

\author{
S. Goto \\ Department of Applied Mathematics and Physics, \\ Graduate School of Informatics, Kyoto University, Kyoto, Japan \\ goto.shinichiro.5r@kyoto-u.ac.jp \\ R. W. Tucker \\ Department of Physics, \\ University of Lancaster and Cockcroft Institute, \\ Daresbury Laboratory, Warrington, UK \\ r.tucker@lancaster.ac.uk \\ T. J. Walton \\ Department of Mathematics, \\ University of Bolton, Deane Campus, Bolton, UK \\ t.walton@bolton.ac.uk
}

Received 10 November 2015

Revised 2 July 2018

Accepted 12 September 2018

Published 4 October 2018

\begin{abstract}
This article explores a number of issues associated with the problem of calculating and detecting electromagnetic quantum induced energy and stress in a stationary dielectric material with a smooth inhomogeneous polarizability. By concentrating on a particular system composed of an ENZ-type (epsilon-near-zero) meta-material, chosen to have a particular anisotropic and smooth inhomogeneous permittivity, confined in an infinitely long perfectly conducting open rectangular waveguide, we are able to deduce analytically from the source-free Maxwell's equations and their boundary conditions a complete set of bounded harmonic electromagnetic evanescent eigen-modes and their associated eigen-frequencies. Since these solutions prohibit the existence of asymptotic scattering states in the guide, the application of the conventional Lifshitz approach to the Casimir stress problem becomes uncertain. An alternative approach is adopted based upon the spectral properties of the system and a regularization scheme constructed with direct applicability to more general systems composed of dielectrics with smooth inhomogeneous permittivities and open systems that may only admit evanescent modes. This more general scheme enables one, for the first time, to prescribe precise criteria for the
\end{abstract}

This is an Open Access article published by World Scientific Publishing Company. It is distributed under the terms of the Creative Commons Attribution 4.0 (CC-BY) License. Further distribution of this work is permitted, provided the original work is properly cited. 
extraction of finite quantum expectation values from regularized mode sums together with error bounds on these values, and is used to derive analytic or numeric results for regularized electromagnetic ground state expectation values in the guide.

Keywords: Casimir; regularization; inhomogeneous dielectric; ENZ metamaterial.

Mathematics Subject Classification 2010: 35Q61, 40A25, 65B15, 78A50, 78M99, 81T55

\section{Introduction}

It is, perhaps, surprising that 70 years since Casimir's prediction [1] of quantum induced electromagnetic forces between a pair of rigid, plane, perfectly conducting, uncharged plates in a material vacuum, there remain many challenging problems in constructing a viable general theory of quantum fluctuation phenomena in continuous dielectric media. With the rise in developments in nanotechnology and the fabrication of artificial dielectrics (meta-materials), such problems deserve scrutiny since their resolution has direct relevance to both technology and our understanding of fundamental aspects of quantum electrodynamics.

One of the earliest attempts to extend Casimir's work to accommodate quantum induced stresses in dielectric media [2] employed the powerful "fluctuationdissipation" approach to calculate the stress between two separated planar half-spaces in the vacuum. It provided an analytic expression for such stresses for dispersive media with piecewise inhomogeneous, lossy permittivity in thermal equilibrium at arbitrary temperatures. The derivation of this expression has since been intensively explored from a number of different starting points and has led to some confusion regarding its universality since differing points of departure often exploited different basic assumptions in their derivations. This has recently led to some authors arguing that the original Lifshitz theory does not have such claimed universality [3]. One might take the attitude that more recent derivations of the Lifshitz formulae render the early derivations obsolete. However, alternative modern derivations 4] are also circumscribed by assumptions that are often implicit and not always mutually compatible.

In common with many methods in quantum field theory a regularization scheme is needed to ameliorate infinities that often arise during the computation of certain physical quantities involving an infinite number of field modes. In general such procedures within a particular scheme lead to the isolation of singularities that are removed to render expressions of interest finite. It remains an open question whether any non-uniqueness of this process invalidates the scheme involved since the final arbiter of all such schemes remains validation of the result by experiment 28]. Furthermore, there is little consensus in the literature on how best to interpret any regularization process that extracts a finite Casimir energy or stress from a physical standpoint. Opinions vary, ranging from regarding Casimir regularization as a matter of definition to interpretations based on physical properties of the systems in interaction. For example, in the computation of finite forces between disjoint open (interacting) continua part of the mathematical procedure is sometimes 
justified as the removal of infinite back reactions of media sub-systems on themselves. Lifshitz used this argument in his original paper [2] and claimed that infinite stresses are "in fact compensated by similar forces at the other side of the body". Without some indication about the mechanical constitutive properties of the media involved, this is a strong statement should any of them sustain inhomogeneous stresses in non-symmetric equilibrium configurations. In addition, certain regularization recipes are sometimes advanced as giving regularization independent results without full justification. While admitting that a number of different schemes appear to yield identical results it is not clear why others that yield different results should a priori be discarded. Since the experimental detection of Casimir forces on non-uniform material (or more generally stresses in dielectric media) is challenging, particularly in the presence of gravitational fields and thermal fluctuations, a precise formulation that underpins each regularization scheme predicting such phenomena is clearly necessary.

To simplify computations, much theoretical work has concentrated on planar [5], spherically or cylindrically symmetric piecewise smooth [6] and piecewise homogeneous dielectric media composed of mechanically rigid sub-structures that respond linearly to only quantum electromagnetic fluctuations [7]. Many recent derivations approach Lifshitz stresses in media by first calculating a regularized energy (or free energy in a thermodynamic context). For systems composed of piecewise homogeneous, isotropic dielectrics, the quantum induced total mechanical pressure across a dielectric interface is then sought by differentiating an integrated expectation value of a regularized interaction energy with respect to a geometrical parameter. However computing a quantum induced stress at any point of a piecewise anisotropic or inhomogeneous dielectric medium with smooth spatially varying permittivities, in general, requires calculating an expectation value of a regularized stress tensor that includes the sum of both electromagnetic and mechanical stresses. In many circumstances, an essential precursor to this analysis is an estimation of the response of the electromagnetic spectral properties of a system to a variation in the geometry and constitutive properties of the components that comprise the system.

Although no natural or fabricated material continuum is strictly inextensible many Casimir calculations on media at rest proceed by assuming that any nonfluidic volume filling dielectrics involved are mechanically rigid (incompressible) and remain static. This inhibits any induced electrostriction effects. However these assumptions imply (even in the absence of quantum induced stresses and inhomogeneous stresses due to the weight of the medium) that the materials involved may be subject to classical mechanical constraints that will result in (possibly localized) classical mechanical stresses (see Sec. 2). Although such mechanical stresses may not influence the electromagnetic spectral problem they are important [8] when it comes to interpreting the results of calculation in order to confront prediction with experiment. For example, in the Lifshitz configuration, both the induced Casimir type stresses that arise in the infinite volume rigid plane separated dielectric half spaces and the constraint induced stresses needed for static equilibrium may be 
different from those in finite volume subsystems with similar dielectric properties used in any laboratory setup.

It has recently been suggested [9] that the Lifshitz prescription can be used to construct the interface stresses for a pair of slabs with piecewise smooth inhomogeneous permittivities by regarding each slab as composed of a large but finite stack of piecewise homogeneous permittivities and then taking the limit as the number in the stack tend to infinity. That this does not in general yield a finite result should come as no surprise since the regularization scheme associated with the reflection coefficients for a finite stack of piecewise homogeneous media is not guaranteed to yield compatible results when using the reflection coefficients associated with the limiting smooth inhomogeneous medium. Numerical evidence supports this assertion. This leaves open the question how best to calculate quantum expectation values of regularized electromagnetic stress tensor components for media with smooth but inhomogeneous permittivities even at zero temperature and without dissipation.

We have argued in [7] that for the original Lifshitz open system, one can proceed without explicit mode regularization and first enclosing such systems in a confining cavity of finite volume and then letting the cavity expand to infinite volume. ${ }^{a}$ The physically allowed quantum states for open media involve mode functions that are regular at all points in space and spatially bounded at infinity. If, furthermore, some propagating modes enable one to construct spatially asymptotic scattering states, one can in principle compute reflection and transmission coefficients, even in the presence of smooth inhomogeneous dielectric media in the system. In the absence of dissipation, one can then attempt to construct an analytic function in the complex angular frequency plane with properties that enable one to discard certain contours in the right-half plane. The singularity structure of the reflection coefficient(s) contains information about the allowed evanescent Maxwell eigen-modes and one may generate (via the Cauchy integral formula) a sum rule relating double integrals over complex functions of real frequency to double integrals of such functions over the imaginary frequency axis. It is this latter double integral that can ultimately be related to expressions proposed by Lifshitz, based on a regularized Maxwell stress tensor in dielectric media. In this approach, when asymptotic propagating modes exist, it is the construction of appropriate analytic functions from the associated reflection coefficients that ensures a viable regularization scheme and yields results equivalent to those found by Lifshitz in his original analysis. In general, however, for media where such scattering states are not present, the traditional Lifshitz approach to the calculation of Casimir stresses in polarizable media is, at best, suspect.

Since all physical systems are in reality open it may appear at first sight that aside from technical complications, the general approach taken by Lifshitz et al. offers, in principle, the only avenue to estimate Casimir type stresses in open systems, including those with spatially varying permittivities. However with the advent

${ }^{a}$ For open systems, one can strictly construct a complete set of orthogonal electromagnetic modes without recourse to a distributional normalization. 
of meta-material technology it is possible to contemplate open dielectric systems that do not permit propagating electromagnetic modes. The absence of scattering states then prohibits any approach based on the use of analytic functions constructed out of asymptotic reflection or transmission coefficients. For such systems an approach based on the construction of regularized expectation values of Maxwell stress tensors offers a possible alternative. In the following such an alternative is discussed in some detail for a particular open waveguide geometry filled with a particular spatially smooth inhomogeneous (non-dispersive) dielectric medium.

This article is organized as follows. Concerning the imposition of mechanical constraints needed to maintain material interfaces in a rigid configuration, Sec. 2 summarizes the conditions involved in maintaining classical static equilibrium in the presence of stationary electromagnetic quantum fluctuations. In Sec. 3, a particular model of a lossless, dispersion free but spatially smooth inhomogeneous dielectric in a perfectly conducting waveguide is introduced that although open, does not admit propagating electromagnetic modes of any frequency. The electromagnetic spectral problem for this system is solved exactly. Section 4 discusses electromagnetic field quantization in a classical dielectric background and the problem of calculating quantum energies and stresses. With the aid of the Euler-Maclaurin summation formula, a consistent regularization scheme is formulated in Sec. 5 to facilitate such calculations and offers, for the first time, a means to reliably extract possible errors in numerical estimates from the remainder terms in the Euler-Maclaurin expansion. In Sec. 6, this summation scheme is verified by evaluating analytically the regularized electromagnetic quantum induced internal energy and stress inside a particular closed perfectly electrically conducting cuboid containing a homogeneous dielectric. This is compared in Sec. 7 to an analytic calculation of the regularized quantum induced energy in a particular open waveguide containing a spatially smooth inhomogeneous dielectric that does not permit propagating electromagnetic modes in the guide. These analytic results are found to be in good agreement with a new numerical scheme based on a subtraction procedure, the interpretation of which is discussed in Sec. 8, In Sec. 9, we summarize our results and conclude that they offer a viable method to estimate quantum induced stresses in systems with smooth inhomogeneous permittivities and a viable numerical regularization scheme with error estimates derived from a generalized Euler-Maclaurin summation.

\section{Continuum Statics}

The detection of electromagnetic Casimir forces on material continua in the laboratory is notoriously difficult due to the competing effects of classical stresses of non-electromagnetic origin. Material containing an inhomogeneous dielectric may also be composed of an inhomogeneous substance with a variable mass density. Its weight in the laboratory may then give rise to additional classical stresses that depend on the orientation and geometry of the material as well as its compressibility and temperature. 
In this section, we summarize the classical Newtonian isothermal balance laws for a static configuration of rigid (incompressible) massive material bodies that can interact with Newtonian gravity and exhibit linear electrical polarizability in external electromagnetic fields. Continuum mechanics in Euclidean 3-space exploits the Killing symmetry of the Euclidean 3-metric at a fundamental level. Thus, in a global Cartesian coordinate system, points $\underline{x}$ in this space can be labeled $\left\{x^{i}\right\}$ with $-\infty<x^{i}<\infty, i=1,2,3$ and in these coordinates, the Euclidean 3-metric tensor field is written

$$
g=\delta_{i j} d x^{i} \otimes d x^{j}
$$

in terms of the Kronecker symbol $\delta_{i j}$ with $i, j=1,2,3$. Furthermore, in these coordinates, the vector fields $\left\{\partial / \partial x^{i}\right\} \equiv\left\{\partial_{i}\right\}$ satisfy $\mathcal{L}_{\partial_{i}} g=0$ and constitute a basis of Killing vector fields for $\mathbb{R}^{3}$. A three-dimensional material body $I$ can be described by a map

$$
\begin{aligned}
\Phi^{I}:[0,1]^{3} & \rightarrow \mathbb{R}^{3} \\
\left(u_{1}, u_{2}, u_{3}\right) & \mapsto \Phi^{I}\left(u_{1}, u_{2}, u_{3}\right)
\end{aligned}
$$

where the parameter domain $[0,1]^{3}$ is conveniently the unit 3 -cube. In $\mathbb{R}^{3}$, a classical material Cauchy stress tensor field on the material body $I$ can be written

$$
\mathcal{G}^{I}=\mathcal{G}_{i j}^{I}(\underline{x}) d x^{i} \otimes d x^{j} .
$$

If $X=X^{i}(\underline{x}) \partial_{i}$ is an arbitrary vector field on $\mathbb{R}^{3}$, we write

$$
\mathcal{G}^{I}(X) \equiv \mathcal{G}_{i j}^{I}(\underline{x}) X^{i}(\underline{x}) d x^{j}
$$

and

$$
\sigma_{X}^{I} \equiv \#\left(\mathcal{G}^{I}(X)\right)=\mathcal{G}_{i j}^{I}(\underline{x}) X^{i}(\underline{x}) \# d x^{j} \equiv \mathcal{G}_{i j}^{I}(\underline{x}) X^{i}(\underline{x}) \varepsilon^{j}{ }_{k l} d x^{k} \wedge d x^{l}
$$

where \# is the Hodge map [10] written here in terms of the Levi-Civita alternating symbol $\varepsilon_{k l}^{j}$. With the aid of the map $\Phi^{I}$, restricting its image in $\mathbb{R}^{3}$ to a surface, any 2 -form $\sigma_{X}^{I}$ can be "pulled back" to any 2-chain parameterizing any two-dimensional surface in $\mathbb{R}^{3}$ and thus facilitates the construction of integrals of stress (or torque density) over arbitrarily shaped surfaces when $X$ generates translations (or rotations relative to any origin).

If a material body does not deform under stress, it is said to be incompressible. In general, properties of incompressibility may reside anywhere throughout the body or in a particular region in the neighborhood of its perimeter. Consider then a region of an isolated body with finite volume $\mathcal{V}^{I}$ and stress tensor $\mathcal{G}^{I}$ bounded by a single closed surface $\mathcal{A}^{I}$. Let $\mathcal{A}^{I}$ be in complete contact with an incompressible medium of volume $\mathcal{V}^{I I}$. Let $\mathcal{V}^{I I}$ be bounded by surfaces $\mathcal{A}^{I}$ and $\mathcal{A}^{I I I}$ where $\mathcal{A}^{I I I}$ is in complete contact with an exterior medium having stress tensor $\mathcal{G}^{I I}$ (see Fig. 1). The stress tensors $\mathcal{G}^{I}$ and $\mathcal{G}^{I I I}$ transmit electromagnetic influences to all regions of $\mathbb{R}^{3}$, but the stress in region $I I$ must be determined from any inextensibility constraints that maintain its rigidity. In addition to the forces transmitted by $\mathcal{G}^{I}$ and $\mathcal{G}^{I I I}$, there are 


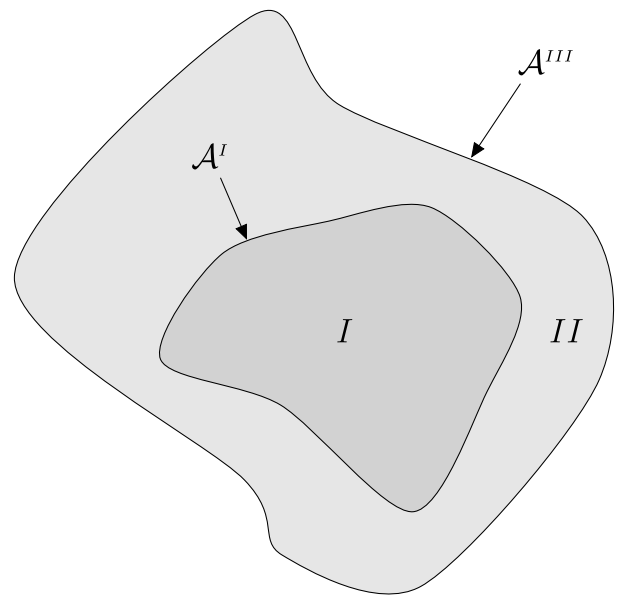

Fig. 1. Three-dimensional domains $I, I I$ and $I I I$ and bounding surfaces $\mathcal{A}^{I}$ and $\mathcal{A}^{I I I}$ referred to in Sec. 2

in general additional "body forces" acting in regions $I, I I$ and $I I I$ due to externally directed fields. These include gravitational forces and externally prescribed forces on the body required to maintain it in static equilibrium with its environment.

The Cartesian component of the total contact force $\mathcal{G}_{i}^{J}\left[\mathcal{A}^{J}\right]$ in direction $\partial_{i}$ is $\int_{\mathcal{A}^{J}} \sigma_{\partial_{i}}^{J}$ for $J \in\{I, I I I\}$. If the body is to remain in static equilibrium under the action of these forces and additional body forces with Cartesian components $\mathcal{B}_{i}^{L}\left[\mathcal{V}^{L}\right]$ for $L \in\{I, I I\}$ together with integrated mechanical forces with Cartesian components $\mathcal{R}_{i}^{I I}\left[\mathcal{V}^{I I}\right]$ that maintain the shapes of $\mathcal{V}^{I}$ and $\mathcal{V}^{I I}$, one has the global static equilibrium conditions

$$
\mathcal{G}_{i}^{I}\left[\mathcal{A}^{I}\right]+\mathcal{G}_{i}^{I I I}\left[\mathcal{A}^{I I I}\right]+\mathcal{B}_{i}^{I}\left[\mathcal{V}^{I}\right]+\mathcal{B}_{i}^{I I}\left[\mathcal{V}^{I I}\right]+\mathcal{R}_{i}^{I I}\left[\mathcal{V}^{I I}\right]=0 \quad \text { for } i=1,2,3
$$

To maintain the static equilibrium of a collection of stationary interacting but isolated bodies of fixed shape, further externally applied stresses are necessary. In general, these additional stresses can be applied in different ways to maintain equilibrium. Such stresses can be implemented by external mechanical forces that are distributed over materials that physically connect the bodies or by external static force fields interacting with them.

Even if one or more of the bodies do not have a finite volume, then the arguments above that require the presence of deformation resisting stresses remain. However, they then apply to any arbitrary finite sub-domain containing an inextensible body with a finite volume and part of its boundary. It is worth noting that in establishing the external stresses required to maintain static equilibrium of a collection of material bodies (whether deformable or not), one stores potential energy that can be released when some or all of the applied constraints are released. If $\mathcal{V}^{I}$ is a domain containing part or all of the volume of a stationary body in the presence of external fields transmitted to it by a stress tensor $\mathcal{G}^{I}$ and body force density $\mathcal{B}^{I}$, 
the integrated force in direction $\partial_{i}$ on $\mathcal{V}^{I}$ is

$$
\mathcal{F}_{i}^{I}\left[\mathcal{V}^{I}\right]=\mathcal{G}_{i}^{I}\left[\partial \mathcal{V}^{I}\right]+\mathcal{B}_{i}^{I}\left[\mathcal{V}^{I}\right]
$$

where $\partial \mathcal{V}^{I}$ denotes the boundary of $\mathcal{V}^{I}$. For a volume $\mathcal{V}^{I}$ containing material with mass density $\rho$ having finite support in $\mathcal{V}^{I}$ immersed in the Earth's gravitational field

$$
\mathcal{B}_{i}^{I}\left[\mathcal{V}^{I}\right]=-g_{0} \int_{\mathcal{V}^{I}} \rho g\left(\partial_{3}, \partial_{i}\right) d x^{1} \wedge d x^{2} \wedge d x^{3} \quad \text { for } i=1,2,3
$$

in terms of the Earth's acceleration of gravity $g_{0}$ and $\partial_{3}$ pointing vertically up from the surface of the Earth. Furthermore, if $\mathcal{G}^{I}$ is smooth on $\mathcal{V}^{I}$

$$
\mathcal{G}_{i}^{I}\left[\partial \mathcal{V}^{I}\right]=\int_{\partial \mathcal{V}^{I}} \sigma_{\partial_{i}}^{I}
$$

In this framework, the 3 -domain $\mathcal{V}^{I}$ is $\Phi^{I}\left([0,1]^{3}\right)$ and $\partial \mathcal{V}^{I}$ is its boundary. It follows from (2.1) that for an isolated body, (2.2) will not be the same as $\int_{\mathcal{A}^{I I I}} \sigma_{\partial_{i}}^{I I I}$, even if one neglects gravitational body forces. Since $\rho>0$, the gravitational body force is strictly only zero if $g_{0}$ is zero. However, even if the body has a dielectric permittivity that depends upon position, the integrated contact force $\mathcal{G}_{i}^{I}\left[\partial \mathcal{V}^{I}\right]$ in an inhomogeneous electromagnetic field is not guaranteed to be always non-zero. These arguments based on classical balance laws lead immediately to:

Theorem 2.1. In the limit when the volume $\mathcal{V}^{I I}$ for an isolated body tends to zero and the forces $\mathcal{B}_{i}^{I I}\left[\mathcal{V}^{I I}\right]+\mathcal{R}_{i}^{I I}\left[\mathcal{V}^{I I}\right]$ tend to an integrated surface traction force component $\mathcal{T}_{i}{ }^{I}\left[\mathcal{A}^{I}\right]$, the static balance conditions become:

$$
\mathcal{G}_{i}^{I}\left[\mathcal{A}^{I}\right]+\mathcal{G}_{i}^{I I I}\left[\mathcal{A}^{I I I}\right]+\mathcal{B}_{i}^{I}\left[\mathcal{V}^{I}\right]+\mathcal{T}_{i}^{I}\left[\mathcal{A}^{I}\right]=0 \quad \text { for } i=1,2,3
$$

where

$$
\mathcal{T}_{i}^{I}\left[\mathcal{A}^{I}\right] \equiv \lim _{\mathcal{V}^{I I} \rightarrow 0}\left(\mathcal{B}_{i}^{I I}\left[\mathcal{V}^{I I}\right]+\mathcal{R}_{i}^{I I}\left[\mathcal{V}^{I I}\right]\right)
$$

and $\mathcal{G}_{i}^{J}\left[\mathcal{A}^{J}\right]$ is the integrated contact force on the area $\mathcal{A}^{J}$ bounding the volume $\mathcal{V}^{J}$ for $J \in\{I, I I, I I I\}$. In this situation, there will exist a non-zero jump $\mathcal{G}_{i}^{I}\left[\alpha^{I}\right]-\mathcal{G}_{i}^{I I}\left[\alpha^{I}\right]$ compatible with (2.3) across any interface $\alpha^{J} \subseteq \mathcal{A}^{J}$.

In most practical cases of relevance, it is precisely at such interfaces where the characteristic properties (such as mass density or permittivity) change discontinuously and where additional information is required in order to match fields across these interfaces. In the context of constructing Casimir forces on a collection of rigid dielectric bodies, one requires a specification of all electromagnetic interface conditions and boundary conditions for global harmonic solutions to Maxwell's equations, together with a Maxwell stress tensor $S^{J}$ for each body in the system. After quantization of the electromagnetic field in the background of a classical dielectric medium, the above balance conditions and definition (2.2) should be used with $\mathcal{G}_{i}^{J}\left[\partial \mathcal{V}^{J}\right]$ replaced by its stationary regularized ground state expectation value for $i=1,2,3$ and each domain of volume $\mathcal{V}^{J}$ in the system. 


\section{Eigenmodes in a Smooth Inhomogeneous Dielectric}

In this section, following the methodology in [5], we construct a complete set of electromagnetic eigen-modes in an infinitely long waveguide of uniform rectangular cross-section filled with a rigid dielectric medium. If the medium has a spatially homogeneous and isotropic permittivity, a waveguide composed of sides with arbitrary conductivity can sustain propagating electromagnetic fields with a continuum of frequencies above a series of mode cutoff frequencies (the frequency cutoff is abrupt - i.e. each harmonic mode has a unique cutoff frequency — if and only if the sides are perfectly conducting) determined by the geometric dimensions of the cross-section and conductivity of the medium and sides of the guide. The same is true for smooth inhomogeneous isotropic media with relative permittivity $\epsilon_{r}>1$ at all points inside the dielectric. However, as will be shown explicitly below, if the medium possesses a positive but smoothly varying inhomogeneous permittivity along the axis of guide, that approaches zero asymptotically along the axis, then in general, this is no longer the case and there may be no propagating modes allowed at any frequency. For such media, all electromagnetic modes have a spatially evanescent behavior along the axis of the guide and a spectrum with discrete frequencies. Such media belong to the class of meta-materials that are sometimes referred to as ENZ dielectrics [1].

To facilitate the following analysis, we consider the idealized case of a dielectric medium without dispersion or absorption and a guide with perfectly conducting, uniform, rectangular cross section. Harmonic fields of angular frequency $\omega$ inside the medium must satisfy the source-free Maxwell equations

$$
\begin{array}{rlrl}
\nabla \times \boldsymbol{E}_{\omega} & =i \omega \boldsymbol{B}_{\omega}, & \nabla \cdot \boldsymbol{B}_{\omega} & =0 \\
\nabla \times \boldsymbol{H}_{\omega} & =-i \omega \boldsymbol{D}_{\omega}, & \nabla \cdot \boldsymbol{D}_{\omega}=0
\end{array}
$$

subject to perfectly conducting boundary conditions on all sides of the guide. The guide axis is chosen to be parallel to the $z$-axis of a Cartesian frame and the sides of the guide are taken to be at $x=0, x=L_{x}, y=0$ and $y=L_{y}$ (see Fig. 2).

Suppose initially that the medium has positive permittivity $\epsilon_{0} \kappa_{1}(z)$, positive permeability $\mu_{0} \kappa_{2}(z)$ and electromagnetic constitutive relations

$$
\boldsymbol{D}_{\omega}=\epsilon_{0} \kappa_{1}(z) \boldsymbol{E}_{\omega} \quad \text { and } \quad \boldsymbol{B}_{\omega}=\mu_{0} \kappa_{2}(z) \boldsymbol{H}_{\omega} .
$$

Since the medium is electrically neutral, one may choose a gauge with harmonic vector potential $\boldsymbol{A}_{\omega}$ such that

$$
\nabla \cdot\left(\kappa_{1}(z) \boldsymbol{A}_{\omega}\right)=0
$$

and

$$
\boldsymbol{E}_{\omega}=i \omega \boldsymbol{A}_{\omega} \quad \text { and } \quad \boldsymbol{B}_{\omega}=\nabla \times \boldsymbol{A}_{\omega}
$$

Then (3.1) are satisfied provided

$$
\frac{1}{\kappa_{1}(z)} \nabla \times\left(\frac{1}{\kappa_{2}(z)} \nabla \times \boldsymbol{A}_{\omega}\right)-\frac{\omega^{2}}{c_{0}^{2}} \boldsymbol{A}_{\omega}=0
$$




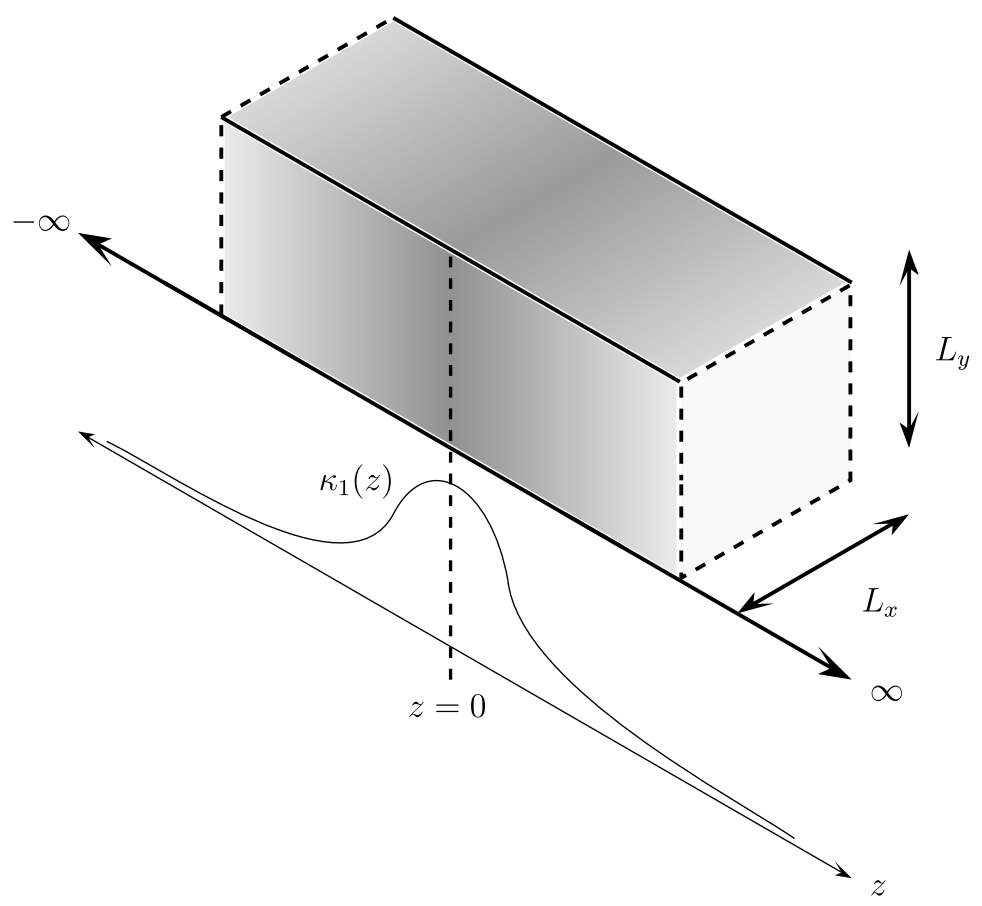

Fig. 2. Geometry of the dielectric waveguide with a smooth inhomogeneous relative permittivity profile $\kappa_{1}(z)=\kappa_{0} \operatorname{sech}^{2}(z / a)$.

where $c_{0}^{2}=1 /\left(\epsilon_{0} \mu_{0}\right)$. Since the interior of the guide is assumed to be simply connected, there are no harmonic TEM modes and the Hodge-De Rham decomposition determines a family of harmonic $T E$ and $T M$ modes in terms of pre-potentials $\mathbf{\Upsilon}_{\omega}^{T E}, \mathbf{\Upsilon}_{\omega}^{T M}$ by the relations

$$
\boldsymbol{A}_{\omega}^{T E}=\frac{1}{\epsilon_{0} \kappa_{1}(z)} \nabla \times \Upsilon_{\omega}^{T E} \quad \text { and } \quad \boldsymbol{A}_{\omega}^{T M}=\frac{1}{\epsilon_{0} \kappa_{1}(z)} \nabla \times\left(\nabla \times \mathbf{\Upsilon}_{\omega}^{T M}\right)
$$

leading to the following result:

Theorem 3.1. The harmonic pre-potentials

$$
\begin{aligned}
& \mathbf{\Upsilon}_{\omega, \mathcal{N}_{s}}^{T E}=\left(0,0, \kappa_{1}(z) \sqrt{\kappa_{2}(z)} f_{\mathcal{N}_{T E}^{T E}}(z) \cos \left(k_{x} x\right) \cos \left(k_{y} y\right)\right) \\
& \boldsymbol{\Upsilon}_{\omega, \mathcal{N}_{s}}^{T M}=\left(0,0, \sqrt{\kappa_{1}(z)} f_{\mathcal{N}_{T M}^{T M}}(z) \sin \left(k_{x} x\right) \sin \left(k_{y} y\right)\right)
\end{aligned}
$$

constitute a basis of solutions with $\mathbf{\Upsilon}_{\omega}^{s}=\sum_{\mathcal{N}_{s}} \mathbf{\Upsilon}_{\omega, \mathcal{N}_{s}}^{s}$ that determine fields that satisfy (3.1) and perfectly conducting boundary conditions at $x=0, x=L_{x}, y=0$ and $y=L_{y}$, provided ${ }^{\mathrm{b}}$

$$
k_{x}=\frac{n_{x} \pi}{L_{x}} \quad \text { and } \quad k_{y}=\frac{n_{y} \pi}{L_{y}} \quad\left(n_{x}, n_{y}=0,1,2, \ldots\right)
$$

${ }^{\mathrm{b}}$ The starting value in the $n_{x}, n_{y}$ range is determined by the existence of non-zero electric and magnetic fields for those values of $n_{x}, n_{y}$, in both the $T E$ and $T M$ sector independently. 
and when the $f_{\mathcal{N}_{s}}^{s}(z)$ are solutions to

$$
\frac{d^{2} f_{\mathcal{N}_{s}}^{s}}{d z^{2}}+\left[\frac{\kappa_{1} \kappa_{2}\left(\omega_{\mathcal{N}_{s}}^{s}\right)^{2}}{c_{0}^{2}}-k_{x}^{2}-k_{y}^{2}+\frac{1}{2 \psi^{s}} \frac{d^{2} \psi^{s}}{d z^{2}}-\frac{3}{\left(2 \psi^{s}\right)^{2}}\left(\frac{d \psi^{s}}{d z}\right)^{2}\right] f_{\mathcal{N}_{s}}^{s}=0
$$

for $s \in\{T E, T M\}$, with

$$
\psi^{T E}=\kappa_{2}(z) \quad \text { and } \quad \psi^{T M}=\kappa_{1}(z) .
$$

Proof. Direct substitution of the harmonic pre-potentials into (3.6) then (3.5).

The basis label $\mathcal{N}_{s}$ appropriate to solutions of (3.9) will be made explicit below (for a particular dielectric medium) and is used to discriminate between distinct physical modes with angular frequency $\omega_{\mathcal{N}_{s}}^{s}$.

All solutions to the differential equation (3.9) that are regular and bounded in the domain $0 \leq x \leq L_{x}, 0 \leq y \leq L_{y},-\infty<z<\infty$ yield physically acceptable solutions defining electromagnetic fields in the open, perfectly conducting guide containing a medium with constitutive properties described by the real, bounded functions $\kappa_{1}(z), \kappa_{2}(z)$. In general, physically acceptable modes with real frequencies can be found that are oscillatory as a function of $z$, as well as being exponentially damped as $|z| \rightarrow \infty$. However, if we choose

$$
\kappa_{1}(z)=\kappa_{0} \operatorname{sech}^{2}\left(\frac{z}{a}\right) \quad \text { and } \quad \kappa_{2}(z)=1
$$

with constants $\kappa_{0}>0, a>0$, then only bounded evanescent modes are found for all frequencies as $z \rightarrow \pm \infty$. In this case, after introducing the dimensionless variables

$$
Z=\frac{z}{a}, \quad \Omega_{\mathcal{N}_{s}}^{s}=\frac{\omega_{\mathcal{N}_{s}}^{s} a \sqrt{\kappa_{0}}}{c_{0}}, \quad \chi=a \sqrt{k_{x}^{2}+k_{y}^{2}}
$$

and writing $\mathcal{Y}_{\mathcal{N}_{s}}^{s}(Z)=f_{\mathcal{N}_{s}}^{s}(z),(3.9)$ becomes

$$
\frac{d^{2} \mathcal{Y}_{\mathcal{N}_{s}}^{s}}{d Z^{2}}+\left(\frac{\left(\Omega_{\mathcal{N}_{s}}^{s}\right)^{2}}{\cosh ^{2}(Z)}-\theta^{s}\right) \mathcal{Y}_{\mathcal{N}_{s}}^{s}=0, \quad s \in\{T E, T M\}
$$

where

$$
\theta^{T E}=\chi^{2} \text { and } \quad \theta^{T M}=\chi^{2}+1 .
$$

It will be shown below that the absence of propagating modes in the guide is a consequence of the positivity of $\theta^{s}$ for both values of $s$. If one ignores the $s$ indices, (3.12) arises in analyzing the physical states for a particle that satisfies the onedimensional Schrödinger equation with a Pöschl-Teller potential well [12] of the form $-U_{0} \operatorname{sech}^{2}(z / a)$. However, aside from the indices $s$, the fundamental difference from (3.12) is that $\theta$ is then the spectral parameter that fixes the particle energy and $\Omega^{2}$ plays the role of the potential strength $U_{0}$. Discrete and continuum physical states are found with $\theta>0$ and $\theta<0$ respectively. In the three-dimensional 
electromagnetic case considered here, the parameter $\theta$ is positive (or zero) due to the imposition of the boundary conditions on the sides of the guide and this is responsible for limiting the allowed states in the waveguide (for both values of $s$ ) to those describing superpositions of only discrete electromagnetic field eigen-modes. For arbitrary $\Omega_{\mathcal{N}_{s}}^{s}, \theta^{s}$ the general solution of (3.12) is expressible in terms of a combination of Gaussian hypergeometric functions (see Appendix A. However, it is non-trivial to isolate from this general representation the physical eigen-solutions (regular and normalizable for all $z$ in the guide) compatible with the constraints $\theta^{s} \geq 0$. A more effective approach for finding the mode eigen-frequencies (and in the process a means to construct the associated eigen-modes) is based on the Frobenius method for solving ordinary differential equations. Since (3.12) is symmetric under $Z \rightarrow-Z$, one may classify solutions as even or odd under this transformation. This leads directly to the following:

Theorem 3.2. For even $\ell=0,2,4, \ldots$ one finds physically acceptable solutions to (3.12) of the form

$$
\mathcal{Y}_{\mathcal{N}_{s}}^{s}(Z)=\frac{1}{\cosh ^{\sqrt{\theta^{s}}}(Z)} \sum_{r=0}^{\ell / 2} C_{\ell, 2 r}^{n_{x}, n_{y}} \cosh ^{-2 r}(Z)
$$

where $\mathcal{N}_{s}=\left\{n_{x}, n_{y}, \ell\right\}$ and the real coefficients $C_{\ell, 2 r}^{n_{x}, n_{y}}$ are all determined in terms of $C_{\ell, 0}^{n_{x}, n_{y}}$ provided

$$
\left(\Omega_{\mathcal{N}_{s}}^{s}\right)^{2}=\left(\ell+\sqrt{\theta^{s}}\right)\left(\ell+\sqrt{\theta^{s}}+1\right)
$$

Similarly, for odd $\ell=1,3,5, \ldots$ one finds solutions to (3.12) of the form

$$
\mathcal{Y}_{\mathcal{N}_{s}}^{s}(Z)=\frac{\sinh (Z)}{\cosh ^{\sqrt{\theta^{s}}}(Z)} \sum_{r=0}^{(\ell-1) / 2} C_{\ell, 2 r+1}^{n_{x}, n_{y}} \cosh ^{-(2 r+1)}(Z)
$$

where the real coefficients $C_{\ell, 2 r+1}^{n_{x}, n_{y}}$ are all determined in terms of $C_{\ell, 1}^{n_{x}, n_{y}}$ provided

$$
\left(\Omega_{\mathcal{N}_{s}}^{s}\right)^{2}=\left(\ell+\sqrt{\theta^{s}}\right)\left(\ell+\sqrt{\theta^{s}}+1\right) .
$$

Proof. See Appendix A.

This is an essential prerequisite in developing our regularization programme below and the fact that, for all $\mathcal{N}_{s}$, these eigenvalues increase monotonically with increasing $\ell$ will play an important role. One may readily verify the following consequence of this theorem:

Corollary 3.1. The $\left\{\mathcal{Y}_{\mathcal{N}_{s}}^{s}(Z)\right\}$ satisfy the orthogonality conditions

$$
\int_{-\infty}^{\infty} \frac{d Z}{\cosh ^{2}(Z)} \mathcal{Y}_{n_{x}, n_{y}, \ell}^{s}(Z) \mathcal{Y}_{n_{x}, n_{y}, \ell^{\prime}}^{s}(Z)=\Lambda_{n_{x}, n_{y}, \ell}^{s} \delta_{\ell \ell^{\prime}}
$$


for constants $\left\{\Lambda_{n_{x}, n_{y}, \ell}^{s}\right\}$ that are determined by a choice of mode normalization. The completeness relation may then be written:

$$
\sum_{\ell} \mathcal{Y}_{n_{x}, n_{y}, \ell}^{s}(Z) \mathcal{Y}_{n_{x}, n_{y}, \ell}^{s}\left(Z^{\prime}\right) / \Lambda_{n_{x}, n_{y}, \ell}^{s}=\cosh ^{2}(Z) \delta\left(Z-Z^{\prime}\right)
$$

Since each $\theta^{s}$ depends uniquely on $n_{x}, n_{y}$ for each $s$, for typographic clarity we can abbreviate $\left\{n_{x}, n_{y}, \ell\right\}$ by $\left\{\theta^{s}, \ell\right\}$ and write $\mathcal{Y}_{n_{x}, n_{y}, \ell}^{s}(Z)=\mathcal{C}_{n_{x}, n_{y}, \ell}^{s} \mathcal{Z}_{\theta^{s}, \ell}^{s}(Z)$ for such a choice. Explicit formulae for these functions are derived in Appendix A and some are displayed in Fig. 3.

A complete set of normalizable pre-potentials is thereby obtained by substituting these solutions into (3.7). The electromagnetic field eigen-modes are then calculated from (3.6) and (3.4). Some of the pre-potential eigen-modes are pure gauge (i.e. they give rise to vanishing electromagnetic fields). If $n_{x}=0$ and $n_{y}=0$, (in which case $\left.\theta^{T E}=0, \theta^{T M}=1\right)$, all field eigen-modes are zero. Furthermore, if $n_{x}=0$ and $n_{y}>0$ or $n_{x}>0$ and $n_{y}=0$, all $T M$ field eigen-modes are zero. It is clear that all time harmonic eigen-modes have spatially oscillatory behavior in directions transverse to the axis of the guide, and exponentially decreasing behavior along the axis of

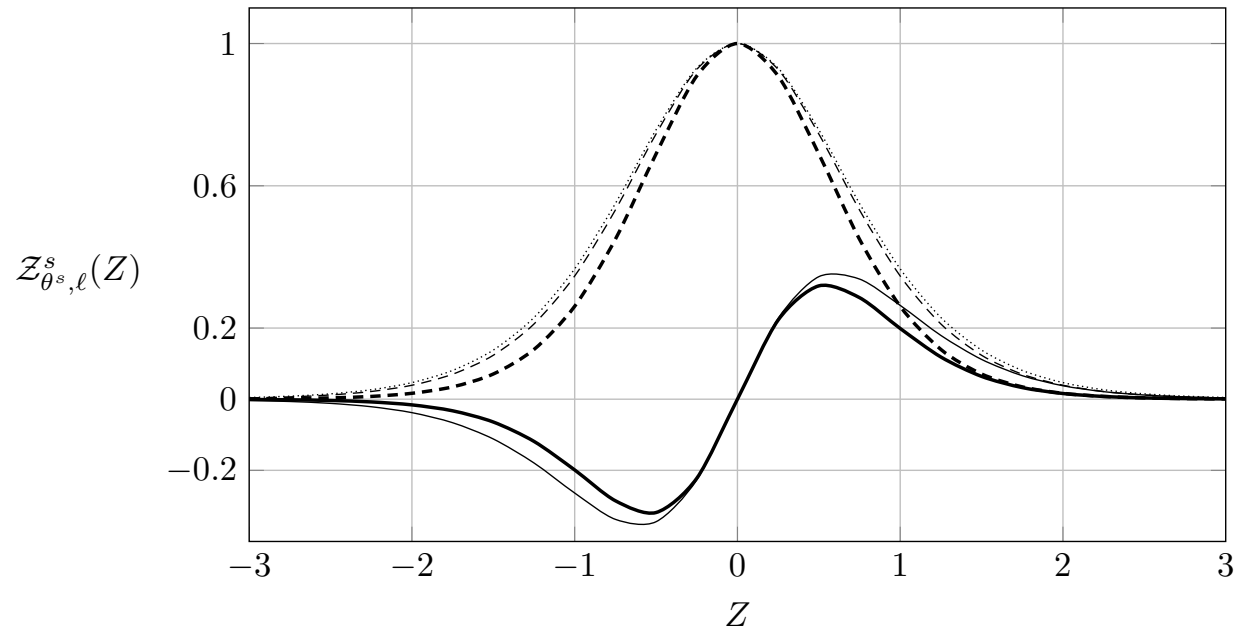

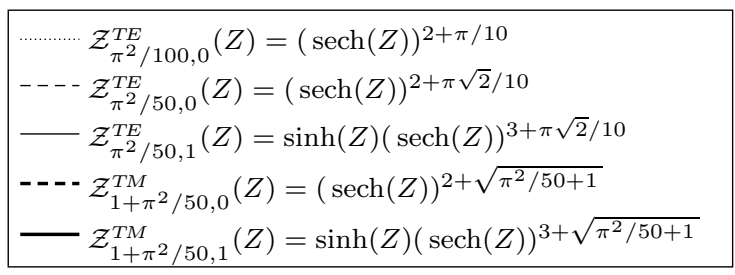

Fig. 3. Behavior of the functions $\mathcal{Z}_{\theta, \ell}^{T E}(Z)$ and $\mathcal{Z}_{\theta, \ell}^{T M}(Z)$ that illustrates the evanescent structure of the five lowest frequency pre-potential eigen-modes in an open guide with a square cross-section containing a medium with relative permittivity $\operatorname{sech}^{2}(Z)$ and unit relative permeability. 
the guide in both directions away from the section at $z=0$ where the permittivity attains its maximum value. In particular, none of the harmonic fields propagate in the guide.

\section{Field Quantization in a Classical Dielectric Background}

Suppose the spectrum $\left\{\omega_{\mathcal{N}_{s}}^{s}\right\}$ of the electromagnetic field modes in the dielectric medium is uniquely labeled by a triple of discrete indices $\mathcal{N}_{s}$ and each associated real eigen-mode is normalized within this domain. Since the complete set of corresponding real field eigen-modes $\boldsymbol{E}_{\omega, \mathcal{N}_{s}}^{s}, \boldsymbol{B}_{\omega, \mathcal{N}_{s}}^{s}$ can be constructed from the harmonic mode pre-potentials, one can introduce Hermitian field operators

$$
\begin{aligned}
\widehat{\boldsymbol{E}}^{s}(x, y, z, t)= & \sum_{\mathcal{N}_{s}}\left(\boldsymbol{E}_{\omega, \mathcal{N}_{s}}^{s}(x, y, z) \widehat{a}_{\mathcal{N}_{s}}^{s \dagger} \exp \left(-i \omega_{\mathcal{N}_{s}}^{s} t\right)\right. \\
& \left.+\boldsymbol{E}_{\omega, \mathcal{N}_{s}}^{s *}(x, y, z) \widehat{a}_{\mathcal{N}_{s}}^{s} \exp \left(i \omega_{\mathcal{N}_{s}}^{s} t\right)\right) \\
\equiv & \sum_{\mathcal{N}_{s}} \widehat{\boldsymbol{E}}_{\omega, \mathcal{N}_{s}}^{s}(x, y, z, t)
\end{aligned}
$$

and

$$
\begin{aligned}
\widehat{\boldsymbol{B}}^{s}(x, y, z, t)= & \sum_{\mathcal{N}_{s}}\left(\boldsymbol{B}_{\omega, \mathcal{N}_{s}}^{s}(x, y, z) \widehat{a}_{\mathcal{N}_{s}}^{\dagger} \exp \left(-i \omega_{\mathcal{N}_{s}}^{s} t\right)\right. \\
& \left.+\boldsymbol{B}_{\omega, \mathcal{N}_{s}}^{s *}(x, y, z) \widehat{a}_{\mathcal{N}_{s}}^{s} \exp \left(-i \omega_{\mathcal{N}_{s}}^{s} t\right)\right) \\
\equiv & \sum_{\mathcal{N}_{s}} \widehat{B}_{\omega, \mathcal{N}_{s}}^{s}(x, y, z, t),
\end{aligned}
$$

in terms of the generators $\widehat{a}_{\mathcal{N}_{s}}^{s}, \widehat{a}_{\mathcal{N}_{s}}^{s}$ of an operator algebra satisfying

$$
\left[\widehat{a}_{\mathcal{N}_{s}}^{s}, \widehat{a}_{\mathcal{N}_{s}^{\prime}}^{s^{\prime} \dagger}\right]=\delta_{\mathcal{N}_{s} \mathcal{N}_{s}^{\prime}} \delta^{s s^{\prime}}
$$

and multi-mode ground states $\left|\Psi^{s}\right\rangle=\bigotimes_{\mathcal{N}_{s}}\left|0_{\mathcal{N}_{s}}\right\rangle$ defined by $\widehat{a}_{\mathcal{N}_{s}}^{s}\left|\Psi^{s}\right\rangle=0$ with normalization $\left\langle 0_{\mathcal{N}_{s}} \mid 0_{\mathcal{N}_{s}}\right\rangle=1$ for all $s, \mathcal{N}_{s}$. These relations permit a construction of a Fock space basis for quantum states of the electromagnetic field. The electromagnetic quantum field theory in the medium is first defined by adopting the Hamiltonian

$$
\sum_{s} \widehat{H}^{s}=\sum_{s, \mathcal{N}_{s}} \int_{\mathcal{V}} \widehat{\mathcal{H}}_{\mathcal{N}_{s}}^{s} d x d y d z
$$

with local (Hermitian) Hamiltonian density $\widehat{\mathcal{H}}_{\mathcal{N}_{s}}^{s}=\left(\widehat{\mathscr{H}}_{\mathcal{N}_{s}}^{s}+\widehat{\mathscr{H}}_{\mathcal{N}_{s}}^{\dagger}\right) / 2$ where

$$
\widehat{\mathscr{H}}_{\mathcal{N}_{s}}^{s}=\frac{1}{2}\left(\widehat{\boldsymbol{E}}_{\omega, \mathcal{N}_{s}}^{s} \cdot \widehat{\boldsymbol{D}}_{\omega, \mathcal{N}_{s}}^{s}+\widehat{\boldsymbol{B}}_{\omega, \mathcal{N}_{s}}^{s} \cdot \widehat{\boldsymbol{H}}_{\omega, \mathcal{N}_{s}}^{s}\right),
$$

and each time-harmonic mode normalized so that

$$
\left\langle\widehat{H}_{\mathcal{N}_{s}}^{s}\right\rangle \equiv\left\langle\Psi^{s}\left|\int_{\mathcal{V}} \widehat{\mathcal{H}}_{\mathcal{N}_{s}}^{s} d x d y d z\right| \Psi^{s}\right\rangle=\frac{1}{2} \hbar \omega_{\mathcal{N}_{s}}^{s}
$$

where $\mathcal{V}$ is the domain containing the dielectric. 
Suppose $\widehat{\mathcal{O}}$ is a local Hermitian operator that has the mode decomposition $\widehat{\mathcal{O}}=$ $\sum_{s, \mathcal{N}_{s}} \widehat{\mathcal{O}}_{\mathcal{N}_{s}}$. For any operator $\widehat{\mathcal{O}}$ acting on states in the Fock space, the expectation value of $\widehat{\mathcal{O}}$ in the general state $\Phi$ :

$$
E_{\Phi}[\widehat{\mathcal{O}}] \equiv\langle\Phi|\widehat{\mathcal{O}}| \Phi\rangle
$$

that includes an infinite number of eigen-modes will rarely converge. In the physical context here this is sometimes ascribed to the neglect of contributions that should be included in order to bring a particular physical system into existence.

Undoubtedly, the most elegant interpretation of the sums (4.3) based on eigensystems is in terms of a complex function $\zeta_{\widehat{\mathcal{O}}}$ of $\sigma \in \mathbb{C}$ associated with the spectrum $\left\{\lambda_{r}\right\}$ of $\widehat{\mathcal{O}}$ and having the representation $\sum_{r} \lambda_{r}^{-\sigma}$ for some $\operatorname{Re}(\sigma)>\sigma_{0}$. The regularized value of (4.3) is then defined as $\zeta_{\widehat{\mathcal{O}}}(-1)$ [13 15. It is often a non-trivial task to determine this value directly from any particular spectrum $\left\{\lambda_{r}\right\}$ and to identify the nature of the singularities of $\zeta_{\widehat{\mathcal{O}}}(\sigma)$ in the complex $\sigma$-plane. Indeed, the analytic properties of a zeta function associated with the spectrum given by (3.14) are not available. This is a prime motivation for our approach: the accommodation of systems with spectra for which an analytic continuation of $\zeta_{\widehat{\mathcal{O}}}(\sigma)$ in $\sigma$ is not known.

Alternative procedures that involve both real and complex analytic continuations or point-splitting schemes offering subtraction processes motivated by physical criteria [16 19] do exist. Casimir's original subtraction scheme 1] was based on the Euler-Maclaurin formula and employed a smooth attenuation map $F_{\sigma}$ in the summation for $\widehat{\mathcal{O}}$ in (4.3) simulating the high-frequency transparency of physically realistic conducting plates. It was then straightforward to show that, for the electromagnetic source-free ground state Hamiltonian $\widehat{H}_{\text {vac }}$ obtained from (4.1) with $\widehat{\boldsymbol{D}}_{\omega, \mathcal{N}_{s}}^{s}=\epsilon_{0} \widehat{\boldsymbol{E}}_{\omega, \mathcal{N}_{s}}^{s}$ and $\widehat{\boldsymbol{B}}_{\omega, \mathcal{N}_{s}}^{s}=\mu_{0} \widehat{\boldsymbol{H}}_{\omega, \mathcal{N}_{s}}^{s}$, the expression

$$
\sum_{s}\left(\sum_{\mathcal{N}_{s}}\left\langle\Psi^{s}\left|\widehat{H}_{\mathrm{vac}, \mathcal{N}_{s}}^{s} F_{\sigma}\left(\widehat{H}_{\mathrm{vac}, \mathcal{N}_{s}}^{s}\right)\right| \Psi^{s}\right\rangle-\int\left\langle\Psi^{s}\left|\widehat{H}_{\mathrm{vac}, \mathcal{N}_{s}}^{s} F_{\sigma}\left(\widehat{H}_{\mathrm{vac}, \mathcal{N}_{s}}^{s}\right)\right| \Psi^{s}\right\rangle d \mathcal{N}^{s}(\Omega)\right)
$$

with $d \mathcal{N}^{s}(\Omega)$ the density of states in the absence of the original Casimir system plates was a convergent power series in the real parameter $\sigma$ characterizing the onset of the high frequency transparency regime. However, the generation of such a series using any such smooth cut-off function is not generic. Whilst it does occur for conducting plates in the vacuum, the presence of a smooth inhomogeneous dielectric medium between the plates does not in general lead to a convergent power series in a physically motivated attenuation parameter. Furthermore, sharp cut-off functions are unphysical and the Euler-Maclaurin approach requires certain differentiability conditions for its implementation.

Based on the Euler-Maclaurin formula, a procedure will be explicitly constructed in Sec. 5 to regularize electromagnetic energy and stress associated with the eigen-system describing the evanescent modes in the previous section. In the 
following, we will also exploit explicit expressions for the normalized states in a perfectly conducting cuboid cavity filled with a homogeneous (non-dispersive) medium of constant relative permittivity.

Theorem 4.1. The cuboid TE pre-potential (3.7) for fields in a perfectly conducting cuboid cavity with rectangular dimensions $L_{x} \times L_{y} \times$ a, filled with a homogenous (non-dispersive) medium of constant relative permittivity $\kappa_{1}=\kappa_{0}>0$ and relative permeability $\kappa_{2}=1$, is given by $f_{\mathcal{N}_{T E}^{T E}}(z)=\mathcal{C}_{\mathcal{N}_{T E}^{T E}}^{T i n}\left(k_{z} z\right)$ where

$$
k_{z}=\frac{\pi n_{z}}{a} \quad\left(n_{z}=1,2,3, \ldots\right),
$$

and $n_{x}, n_{y}=0,1,2, \ldots$ with $n_{x} \neq n_{y}=0$. The associated angular frequency eigenvalue $\omega_{\underline{\mathcal{N}}}^{T E}$ is given by:

$$
\left(\Omega_{\underline{\mathcal{N}}}^{T E}\right)^{2} \equiv \frac{\left(\omega_{\underline{\mathcal{N}}}^{T E}\right)^{2} a^{2} \kappa_{0}}{c_{0}^{2}}=a^{2}\left(k_{x}^{2}+k_{y}^{2}+k_{z}^{2}\right)
$$

where $\underline{\mathcal{N}}=\left\{n_{x}, n_{y}, n_{z}\right\}$ and

$$
\left(\mathcal{C}_{\underline{\mathcal{N}}}^{T E}\right)^{2}=\frac{4 \hbar \epsilon_{0} \eta^{T E}}{\kappa_{0} L_{x} L_{y} a\left(k_{x}^{2}+k_{y}^{2}\right) \omega_{\underline{\mathcal{N}}}^{T E}}
$$

where

$$
\eta^{T E}=\left\{\begin{array}{l}
1, \quad \text { for } n_{x}, n_{y} \geq 1 \\
\frac{1}{2}, \quad \text { for } n_{x}=0 \text { or } n_{y}=0
\end{array}\right.
$$

Similarly, the cuboid TM pre-potential follows with $f_{\mathcal{N}_{T M}^{T M}}(z)=\mathcal{C}_{\mathcal{N}_{T M}^{T M}} \cos \left(k_{z} z\right)$ where

$$
k_{z}=\frac{\pi n_{z}}{a} \quad\left(n_{z}=0,1,2, \ldots\right)
$$

and $n_{x}, n_{y}=1,2, \ldots$ The associated angular frequency eigenvalue $\omega_{\underline{\mathcal{N}}}^{T M}$ is given by:

$$
\left(\Omega_{\underline{\underline{\mathcal{N}}}}^{T M}\right)^{2} \equiv \frac{\left(\omega_{\underline{\mathcal{N}}}^{T M}\right)^{2} a^{2} \kappa_{0}}{c_{0}^{2}}=a^{2}\left(k_{x}^{2}+k_{y}^{2}+k_{z}^{2}\right)
$$

and

$$
\left(\mathcal{C}_{\underline{\mathcal{N}}}^{T M}\right)^{2}=\frac{4 \hbar c_{0}^{2} \epsilon_{0} \eta^{T M}}{\kappa_{0} L_{x} L_{y} a\left(k_{x}^{2}+k_{y}^{2}\right)\left(\omega_{\underline{\mathcal{N}}}^{T M}\right)^{3}}
$$

where

$$
\eta^{T M}= \begin{cases}1, & \text { for } n_{z} \geq 1 \\ \frac{1}{2}, & \text { for } n_{z}=0\end{cases}
$$


Proof. For the TE pre-potential, the modes with $n_{z}=0$ and the modes with $n_{x}=n_{y}=0$ do not contribute to the fields in the system since they are pure gauge modes. Hence, from Theorem 3.1 .

$$
\boldsymbol{\Upsilon}_{\omega}^{T E}=\sum_{n_{x}=1}^{\infty} \sum_{n_{y}=1}^{\infty} \sum_{n_{z}=1}^{\infty} \boldsymbol{\Upsilon}_{\omega, \underline{\mathcal{N}}}^{T E}+\sum_{n_{x}=1}^{\infty} \sum_{n_{z}=1}^{\infty} \boldsymbol{\Upsilon}_{\omega, n_{x}, 0, n_{z}}^{T E}+\sum_{n_{y}=1}^{\infty} \sum_{n_{z}=1}^{\infty} \boldsymbol{\Upsilon}_{\omega, 0, n_{y}, n_{z}}^{T E}
$$

where

$$
\mathbf{\Upsilon}_{\omega, \underline{\mathcal{N}}}^{T E} \equiv \mathbf{\Upsilon}_{\omega, n_{x}, n_{y}, n_{z}}^{T E}=\left(0,0, \kappa_{0} \mathcal{C}_{\underline{\mathcal{N}}}^{T E} \sin \left(k_{z} z\right) \cos \left(k_{x} x\right) \cos \left(k_{y} y\right)\right)
$$

with normalization constants $\left\{\mathcal{C}_{\underline{\underline{\mathcal{N}}}}^{T E}\right\}$. This pre-potential generates fields that satisfy the appropriate boundary conditions on the cavity boundary and determines (4.4) for the angular frequency $\omega_{\underline{\underline{\mathcal{N}}}}^{T E}$. Furthermore, one may verify (4.5) for the normalization constants $\left\{\mathcal{C}_{\underline{\mathcal{N}}}^{T E}\right\}$ using (3.2), (3.4), (3.6) and (4.2) for this pre-potential.

Similarly for the cuboid $T M$ pre-potential, the modes with $n_{x}=0$ and the modes with $n_{y}=0$ do not contribute to the fields in the system since they are pure gauge modes, yielding:

$$
\mathbf{\Upsilon}_{\omega}^{T M}=\sum_{n_{x}=1}^{\infty} \sum_{n_{y}=1}^{\infty} \sum_{n_{z}=0}^{\infty} \mathbf{\Upsilon}_{\omega, \underline{\mathcal{N}}}^{T M}
$$

where

$$
\mathbf{\Upsilon}_{\omega, \underline{\mathcal{N}}}^{T M}=\left(0,0, \sqrt{\kappa_{0}} \mathcal{C}_{\underline{\underline{\mathcal{N}}}}^{T M} \cos \left(k_{z} z\right) \sin \left(k_{x} x\right) \sin \left(k_{y} y\right)\right)
$$

in terms of the normalization constants $\left\{\mathcal{C}_{\underline{\mathcal{N}}}^{T M}\right\}$. This pre-potential generates fields that satisfy the appropriate boundary conditions on the cavity boundary and determines (4.6) for the angular frequency $\omega_{\underline{\mathcal{N}}}^{T M}$. One readily verifies (4.7) using (3.2), (3.4), (3.6) and (4.2) for this pre-potential.

In the following, we also require explicit expressions for the normalized states in the open guide containing the inhomogeneous dielectric (3.10) and for the general ground state expectation value of the electromagnetic energy and stress operator:

Theorem 4.2. With $\underline{\mathcal{N}}=\left\{n_{x}, n_{y}, \ell\right\}$, the ground state expectation values of the Hamiltonian densities $\widehat{\widehat{H}}_{\underline{\mathcal{N}}}^{s}$ for the system containing the inhomogeneous dielectric (3.10) are given by:

$$
\begin{aligned}
& \left\langle\widehat{H}_{\underline{\mathcal{N}}}^{T E}\right\rangle=\frac{\left(\mathcal{C}_{\underline{\underline{\mathcal{N}}}}^{T E}\right)^{2}\left(\omega_{\underline{\underline{\mathcal{N}}}}^{T E}\right)^{2} \kappa_{0} L_{x} L_{y}\left(k_{x}^{2}+k_{y}^{2}\right)}{4 \epsilon_{0} \eta^{T E}} I_{\underline{\mathcal{N}}}^{T E}, \\
& \left\langle\widehat{H}_{\underline{\mathcal{N}}}^{T M}\right\rangle=\frac{\left(\mathcal{C}_{\underline{\mathcal{N}}}^{T M}\right)^{2}\left(\omega_{\underline{\underline{\mathcal{N}}}}^{T M}\right)^{4} \kappa_{0} L_{x} L_{y}\left(k_{x}^{2}+k_{y}^{2}\right)}{4 \epsilon_{0} c_{0}^{2}} I_{\underline{\mathcal{N}}}^{T M}
\end{aligned}
$$

where

$$
I_{\underline{\mathcal{N}}}^{s} \equiv \int_{-\infty}^{\infty} \frac{d Z}{\cosh ^{2}(Z)}\left(\mathcal{Z}_{\ell, \theta^{s}}(Z)\right)^{2}
$$


The normalization constants $\left\{\mathcal{C}_{\underline{\mathcal{N}}}^{s}\right\}$ are then explicitly given in terms of these convergent integrals:

$$
\begin{aligned}
\left(\mathcal{C}_{\underline{\mathcal{N}}}^{T E}\right)^{2} & =\frac{2 \hbar \epsilon_{0} \eta^{T E}}{\kappa_{0} L_{x} L_{y}\left(k_{x}^{2}+k_{y}^{2}\right) \omega_{\underline{\mathcal{N}}}^{T E} I_{\underline{\mathcal{N}}}^{T E}}, \\
\left(\mathcal{C}_{\underline{\mathcal{N}}}^{T M}\right)^{2} & =\frac{2 \hbar \epsilon_{0} c_{0}^{2}}{\kappa_{0} L_{x} L_{y}\left(k_{x}^{2}+k_{y}^{2}\right)\left(\omega_{\underline{\mathcal{N}}}^{T M}\right)^{3} I_{\underline{\mathcal{N}}}^{T M}} .
\end{aligned}
$$

Proof. Using Corollary 3.1 and calculations in Appendix A, these follow directly from the normalization condition (4.2).

To compute the induced Casimir force (or torque) expectation values in any state on any surface in $\mathcal{V}$, one requires a Hermitian operator-valued electromagnetic stress tensor in the medium. A natural choice for a medium at rest can be constructed from the classical symmetrized Minkowski Maxwell electromagnetic stress tensor with Cartesian components [22, 23]:

$$
S_{i j}(x, y, z)=-\frac{1}{2}\left(E_{i} D_{j}+D_{i} E_{j}+B_{i} H_{j}+H_{i} B_{j}\right)+\frac{1}{2}\left(E_{k} D^{k}+B_{k} H^{k}\right) \delta_{i j}
$$

and for media with constitutive properties given by (3.10):

$$
\boldsymbol{D}=\epsilon_{0} \kappa_{1}(z) \boldsymbol{E} \quad \text { and } \quad \boldsymbol{B}=\mu_{0} \boldsymbol{H} .
$$

Replacing the classical fields $\boldsymbol{E}, \boldsymbol{B}, \boldsymbol{D}, \boldsymbol{H}$ by the operator mode expansions $\widehat{\boldsymbol{E}}^{s}, \widehat{\boldsymbol{B}}^{s}, \widehat{\boldsymbol{D}}^{s}, \widehat{\boldsymbol{H}}^{s}$ yields for the ground state expectation values of the stress operator tensor components:

$$
\left\langle\widehat{S}_{i j}^{s}(x, y, z)\right\rangle \equiv \sum_{\mathcal{N}_{s}}\left\langle\Psi^{s}\left|\left(\widehat{S}_{\mathcal{N}_{s}}^{s}\right)_{i j}(x, y, z, t)\right| \Psi^{s}\right\rangle \equiv \sum_{\mathcal{N}_{s}}\left\langle\left(\widehat{S}_{\mathcal{N}_{s}}^{s}\right)_{i j}\right\rangle(x, y, z)
$$

with

$$
\begin{aligned}
\left(\widehat{S}_{\mathcal{N}_{s}}^{s}\right)_{i j}= & -\frac{1}{2}\left[\left(\widehat{\boldsymbol{E}}_{\omega, \mathcal{N}_{s}}^{s}\right)_{i}\left(\widehat{\boldsymbol{D}}_{\omega, \mathcal{N}_{s}}^{s}\right)_{j}+\left(\widehat{\boldsymbol{D}}_{\omega, \mathcal{N}_{s}}^{s}\right)_{i}\left(\widehat{\boldsymbol{E}}_{\omega, \mathcal{N}_{s}}^{s}\right)_{j}\right] \\
& -\frac{1}{2}\left[\left(\widehat{\boldsymbol{B}}_{\omega, \mathcal{N}_{s}}^{s}\right)_{i}\left(\widehat{\boldsymbol{H}}_{\omega, \mathcal{N}_{s}}^{s}\right)_{j}+\left(\widehat{\boldsymbol{H}}_{\omega, \mathcal{N}_{s}}^{s}\right)_{i}\left(\widehat{\boldsymbol{B}}_{\omega, \mathcal{N}_{s}}^{s}\right)_{j}\right] \\
& +\frac{1}{2}\left[\left(\widehat{\boldsymbol{E}}_{\omega, \mathcal{N}_{s}}^{s}\right)_{k}\left(\widehat{\boldsymbol{D}}_{\omega, \mathcal{N}_{s}}^{s}\right)^{k}+\left(\widehat{\boldsymbol{B}}_{\omega, \mathcal{N}_{s}}^{s}\right)_{k}\left(\widehat{\boldsymbol{H}}_{\omega, \mathcal{N}_{s}}^{s}\right)^{k}\right] \delta_{i j} .
\end{aligned}
$$

Note that in these expressions, the electromagnetic fields and hence $\left(\widehat{S}_{\mathcal{N}_{s}}^{s}\right)_{i j}$ are all real-valued functions on the domain containing the dielectric.

\section{Regularization}

If the frequency of the electromagnetic normal modes of a system increases indefinitely as a function of the number of zeroes associated with the modes then the ground state energy of each mode will also increase indefinitely. In 
such circumstances, the series in (4.11) will, in general, diverge. A mathematical regularization process is then required to extract a finite value for expectation values. As noted in the introduction, all computations of Casimir stresses involve, either explicitly or implicitly, such a process. It was also noted that applications in the recent literature of Lifshitz methods to certain problems involving smooth inhomogeneous permittivities did not lead to finite stress fields in certain uncharged systems. Furthermore, the absence of scattering states constructed from modes in a certain meta-material prohibits the traditional application of Lifshitz methods, employing reflection and non-zero transmission coefficients, in determining any quantum induced stress in a dielectric medium.

Interpretations of some regularization processes are sometimes motivated by the notion that only energy differences are physical [4, 24] and that perfectly conducting confining surfaces are idealizations that should be replaced by physical interfaces between media that exhibit dispersion with absorption. Approaches along these lines are to be found in 25, 18. Implicit in such methods are regularization processes based on subtraction schemes that involve a suitable Green's function for the system under consideration. Such functions can be constructed from an analysis of the spectral properties of a complete set of solutions to a particular Maxwell boundaryvalue problem.

Many regularization processes require the introduction of an auxiliary expression that is a function of an auxiliary variable defined on the real line or the complex plane. The process proceeds by recognizing the presence of singularities of this auxiliary function in the auxiliary variable. If this is possible the function can then be either analytically continued from its original domain of definition to any chosen value of the auxiliary variable where the continued function is finite or rendered finite by explicitly removing its singular parts [4].

In the quantum description of the waveguide system above, emphasis is on the electromagnetic ground state energy (derived from a choice of Hamiltonian density) and components of a local electromagnetic ground state stress tensor (derived from a choice of electromagnetic stress tensor field). For general systems composed of piecewise spatially homogeneous media, it is possible to relate certain integrals of the latter (total pressures) to derivatives with respect to geometric parameters in the expression for the former. However for systems composed of smooth inhomogeneous media this is not in general possible. In such systems, the electromagnetic ground state energy contributes to the total global internal (free) energy of the system while the local electromagnetic ground state stress tensor components can be used to calculate contributions to the local stresses and average forces at points and surfaces respectively in the medium. Regularization of an energy density expectation value requires a knowledge of the electromagnetic eigen-mode frequencies as a function of system parameters while regularization of a stress expectation value requires, either explicitly or implicitly, a knowledge of both the mode frequencies and a complete set of mode eigen-functions for the system. This is tantamount to a knowledge of the appropriate classical electromagnetic Green's function for the system. 
Since we have a complete set of real solutions to the Maxwell boundary-value problem for a smooth inhomogeneous, non-dispersive ENZ type material, we establish in this section a general real analytic regularization scheme based on the venerable Euler-Maclaurin summation formula [21, 26]. This will be used in subsequent sections to explicitly demonstrate the regularization of quantum expectation values in the context of a simple homogeneous system and that certain derivatives of the global regularized energy density expectation value coincide with the regularized pressure on a confining surface. It is then used to calculate analytically the quantum contribution to the global regularized internal energy of the open waveguide system containing the smooth inhomogeneous dielectric medium described in Sec. 3 as well as outlining how the average quantum induced force on either side of an arbitrary cross-section in the guide follows from an explicit knowledge of the electromagnetic eigen-modes in the medium.

Theorem 5.1 (The Euler-Maclaurin Identity). For any finite integer $n \geq 0$, $m \in \mathbb{Z}^{+}$and any $(2 m+1)$-differentiable map $f: \mathcal{I} \subset \mathbb{R} \rightarrow \mathbb{R}$ with $[n, N] \subset \mathcal{I}$ :

$$
\begin{aligned}
\sum_{k=n}^{N} f(k)-\int_{n}^{N} f(x) d x= & \frac{1}{2}(f(n)+f(N)) \\
& +\sum_{r=1}^{m} \frac{B_{2 r}}{(2 r) !}\left(f^{(2 r-1)}(N)-f^{(2 r-1)}(n)\right) \\
& +\frac{1}{(2 m+1) !} \int_{n}^{N} P_{2 m+1}(x) f^{(2 m+1)}(x) d x
\end{aligned}
$$

where $\left\{B_{k}\right\}$ denotes the set of Bernoulli numbers, $\left\{P_{k}(x)\right\}=\left\{\widetilde{B}_{k}(x-\lfloor x\rfloor)\right\}$ denotes the set of periodic functions associated with the Bernoulli polynomials $\left\{\widetilde{B}_{k}(x)\right\}$ and the integer $N$ satisfies $N>n \geq 0$.

Proof. See [20].

For real-valued functions $f$ satisfying the criteria of the theorem above, the freedom to start the summations on the left above at $k=n$ and $x=n$ for any $n \geq 0$ can often be exploited to facilitate the evaluation of limits that involve the derivatives of $f$ in the subsequent analysis. Following Hardy [20], we introduce the abbreviations:

$$
\begin{aligned}
S_{m}(k)[f] & =\sum_{r=1}^{m} \frac{B_{2 r}}{(2 r) !} f^{(2 r-1)}(k) \\
T_{m}(n, N)[f] & =\frac{1}{(2 m+1) !} \int_{n}^{N} P_{2 m+1}(x) f^{(2 m+1)}(x) d x .
\end{aligned}
$$

Then, since

$$
T_{m}(n, N)[f]=T_{m}(n, \infty)[f]-T_{m}(N, \infty)[f],
$$


the Euler-Maclaurin formula can be rewritten

$$
\sum_{k=n}^{N} f(k)-\int_{n}^{N} f(x) d x=\frac{1}{2} f(N)+S_{m}(N)[f]-T_{m}(N, \infty)[f]+C_{n, m}[f]
$$

where

$$
C_{n, m}[f] \equiv \frac{1}{2} f(n)-S_{m}(n)[f]+T_{m}(n, \infty)[f] .
$$

It should be stressed that (5.3), involving no limiting operations, is an identity for any admissible finite values of $n, N, m$ provided all terms in (5.3) exist. In general, the formula will break down if one lets $m \rightarrow \infty$ for fixed $N$. If, however, $f(x)$ is such that for some finite integer $m \geq 1$ :

$$
\int_{n}^{\infty}\left|f^{(2 m+1)}(x)\right| d x<\infty
$$

then, since $\lim _{N \rightarrow \infty} T_{m}(N, \infty)[f]=0$ as $N \rightarrow \infty$,

$$
\sum_{k=n}^{N} f(k)-\int_{n}^{N} f(x) d x-\frac{1}{2} f(N)-S_{m}(N)[f] \rightarrow C_{n, m}[f] .
$$

If furthermore for some integer $M \geq 1$, (5.5) remains valid for all $m \geq M$ then $\lim _{N \rightarrow \infty}\left(S_{M+1}(N)[f]-S_{M}(N)[f]\right)=0$ which implies $C_{n, m}[f]$ is independent of $m$ for $m \geq M$.

In summary, this leads to the following:

Theorem 5.2 (The Euler-Maclaurin Summation Formula). For any finite integer $n \geq 0, m, M \in \mathbb{Z}^{+}$and any $(2 m+1)$-differentiable map $f: \mathcal{I} \subset \mathbb{R} \rightarrow \mathbb{R}$ with $[n, \infty) \subset \mathcal{I}$, if

$$
\int_{n}^{\infty}\left|f^{(2 m+1)}(x)\right| d x<\infty
$$

is satisfied for all $m \geq M$, in the $N \rightarrow \infty$ limit:

$$
\sum_{k=n}^{\infty} f(k)-\int_{n}^{\infty} f(x) d x-\lim _{N \rightarrow \infty}\left\{\frac{1}{2} f(N)+S_{M}(N)[f]\right\}=C_{n, M}[f]
$$

where $S_{M}(N)[f]$ and $C_{n, M}[f]$ are given by (5.2) and (5.4) respectively.

The finite number $C_{n, M}[f]$ is the Hardy-Ramanujan value (p. 327, [20]) assigned to the "divergent series" $\sum_{k=n}^{\infty} f(k)$. Based on these arguments the existence of a finite $C_{n, M}[f]$ depends critically on $f(x)$ satisfying (5.5) for all integers $m \geq M$.

For the application to Casimir problems in three dimensions, we encounter summations indexed by a triple $\underline{\mathcal{N}}$. It proves expedient to replace these by a simpler, single-indexed summation with integral summands obtained in the limit when $L_{x}, L_{y}$ become large compared with a particular scale $a$. In order for the integrals in this summation to exist, we shall introduce a one-parameter family of (regulator) maps $\left\{f_{\sigma}\right\}$ depending smoothly upon a real dimensionless parameter $\sigma \geq 0$, each of 
which satisfy the criteria of the Euler-Maclaurin identity. Furthermore, we explore conditions on $f_{\sigma}$ that enable one to assign a finite value to the "divergent series" $\sum_{k=n}^{\infty} f_{\sigma}(k)$ for all $\sigma$, generalizing the Euler-Maclaurin summation formula.

To this end, introduce the mapping $\sigma \mapsto \Gamma_{m}^{n}\left[f_{\sigma}\right]$ with

$$
\Gamma_{m}^{n}\left[f_{\sigma}\right] \equiv \frac{1}{2} f_{\sigma}(n)-S_{m}(n)\left[f_{\sigma}\right]+\int_{n}^{\infty} f_{\sigma}(x) d x
$$

and split it into the terms

$$
\Gamma_{m}^{n}\left[f_{\sigma}\right]=\mathcal{P}_{m}^{n}\left[f_{\sigma}\right]+\sum_{j=0}^{\infty} \beta_{m, j}^{n}\left[f_{\sigma}\right] \sigma^{j}
$$

where $\mathcal{P}_{m}^{n}\left[f_{\sigma}\right]$ contains any terms in $\Gamma_{m}^{n}\left[f_{\sigma}\right]$ that are singular as $\sigma$ tends to zero from above. In terms of the (non-unique) decomposition (5.8), in the limit as $N \rightarrow \infty$ the Euler-Maclaurin identity (5.1) can be recast into the form

$$
\begin{gathered}
\sum_{k=n}^{\infty} f_{\sigma}(k)-\mathcal{P}_{m}^{n}\left[f_{\sigma}\right]-\lim _{N \rightarrow \infty}\left(\frac{1}{2} f_{\sigma}(N)+S_{m}(N)\left[f_{\sigma}\right]\right) \\
=T_{m}(n, \infty)\left[f_{\sigma}\right]+\sum_{j=0}^{\infty} \beta_{m, j}^{n}\left[f_{\sigma}\right] \sigma^{j}
\end{gathered}
$$

for any $n \geq 0$. If furthermore for some integer $M \geq 1$,

$$
\int_{n}^{\infty}\left|f_{\sigma}^{(2 m+1)}(x)\right| d x<\infty \text { for all } \sigma \geq 0
$$

remains valid for all $m \geq M$ then $\lim _{N \rightarrow \infty}\left(S_{M+1}(N)\left[f_{\sigma}\right]-S_{M}(N)\left[f_{\sigma}\right]\right)=0$ which implies the right-hand side of (5.9) is independent of $m$ for $m \geq M$. Thus with the above definitions, we arrive at the following generalization of Euler-Maclaurin summation:

Theorem 5.3 (A Generalized Euler-Maclaurin Summation Formula). Let $\left\{f_{\sigma}\right\}$ denote a one-parameter family of maps depending smoothly upon a real dimensionless parameter $\sigma \geq 0$, each of which satisfy the criteria of the Euler-Maclaurin summation formula. Then

$$
\begin{gathered}
\sum_{k=n}^{\infty} f_{\sigma}(k)-\mathcal{P}_{M}^{n}\left[f_{\sigma}\right]-\lim _{N \rightarrow \infty}\left(\frac{1}{2} f_{\sigma}(N)+S_{M}(N)\left[f_{\sigma}\right]\right) \\
=T_{M}(n, \infty)\left[f_{\sigma}\right]+\sum_{j=0}^{\infty} \beta_{M, j}^{n}\left[f_{\sigma}\right] \sigma^{j}
\end{gathered}
$$

for all $n \geq 0, \sigma \geq 0$ where $\mathcal{P}_{M}^{n}\left[f_{\sigma}\right], \beta_{M, j}^{n}\left[f_{\sigma}\right]$ are defined through (5.8), and $S_{M}(N)\left[f_{\sigma}\right], T_{M}(n, \infty)\left[f_{\sigma}\right]$ and defined through (5.2). In the limit $\sigma \rightarrow 0^{+}$, this takes the form:

$$
\lim _{\sigma \rightarrow 0^{+}}\left(\sum_{k=n}^{\infty} f_{\sigma}(k)-\mathcal{P}_{M}^{n}\left[f_{\sigma}\right]-\lim _{N \rightarrow \infty}\left\{\frac{1}{2} f_{\sigma}(N)+S_{M}(N)\left[f_{\sigma}\right]\right\}\right)=T_{M}^{n}+\beta_{M}^{n}
$$


where

$$
T_{M}^{n}=\lim _{\sigma \rightarrow 0^{+}} T_{M}(n, \infty)\left[f_{\sigma}\right] \quad \text { and } \quad \beta_{M}^{n} \equiv \lim _{\sigma \rightarrow 0^{+}} \sum_{j=0}^{\infty} \beta_{M, j}^{n}\left[f_{\sigma}\right] \sigma^{j}=\beta_{M, 0}^{n}\left[f_{\sigma}\right] .
$$

This theorem is central to our construction of a regularization procedure and along with the definitions above, immediately yields the following practical result:

Corollary 5.1. For all real-valued functions $\left\{f_{\sigma}\right\}$ satisfying the criteria of the generalized Euler-Maclaurin summation formula:

$$
\beta_{M}^{n}=\lim _{\sigma \rightarrow 0^{+}}\left(\Gamma_{M}^{n}\left[f_{\sigma}\right]-\mathcal{P}_{M}^{n}\left[f_{\sigma}\right]\right)
$$

For the applications in the following, the real-valued function $f_{\sigma}$ will be chosen so that $\lim _{N \rightarrow \infty} f_{\sigma}(N)=0$ and $\lim _{N \rightarrow \infty} S_{M}(N)\left[f_{\sigma}\right]=0$, yielding

$$
\lim _{\sigma \rightarrow 0^{+}}\left(\sum_{k=n}^{\infty} f_{\sigma}(k)-\mathcal{P}_{M}^{n}\left[f_{\sigma}\right]\right)=T_{M}^{n}+\beta_{M}^{n} .
$$

Furthermore, it will be shown that, while $\beta_{M}^{n}$ may be amenable to exact analytical evaluation, in general, $T_{M}(n, \infty)\left[f_{\sigma}\right]$ is not. However, it follows from the Fourier series representation of the periodic Bernoulli functions [27] that they are bounded:

$$
\left|P_{k}(x)\right| \leq \frac{2 k ! \zeta_{\mathrm{R}}(k)}{(2 \pi)^{k}}
$$

where $\zeta_{\mathrm{R}}(k)$ denotes the Riemann zeta function. Hence, the $T_{m}(n, \infty)\left[f_{\sigma}\right]$ satisfy

$$
\left|T_{m}(n, \infty)\left[f_{\sigma}\right]\right| \leq \varepsilon_{m}^{n}\left[f_{\sigma}\right] \equiv \frac{2 \zeta_{\mathrm{R}}(2 m+1)}{(2 \pi)^{2 m+1}} \int_{n}^{\infty}\left|f_{\sigma}^{(2 m+1)}(x)\right| d x
$$

for all $n, m, \sigma \geq 0$. Thus, from (5.10), the integral in (5.14) is finite and bounded, and for any $n, m, \sigma$ one may readily calculate the value of this bound. Although the magnitude of this bound in the limit $\sigma \rightarrow 0^{+}$will, in general, vary in a complicated manner as a function of $M$, one may verify that for particular values of $M$, the bound may be significantly smaller in magnitude than $\left|\beta_{M}^{n}\right|$. This observation enables one to select $M$ for an asymptotic approximation of the left-hand side of (5.13), regarded as a function of $M$. Consequently, in the following we refer to this bound as an "error term".

Provided, for fixed values of $n, m$, the term $S_{m}(n)\left[f_{\sigma}\right]$ exists as $\sigma \rightarrow 0^{+}$, the relation (5.13) shows how the singularities of $\sum_{k=n}^{\infty} f_{\sigma}(k)$ can be compensated by singularities in the other terms on its left-hand side, since the terms on the righthand side are finite as $\sigma \rightarrow 0^{+}$. This corresponds to a viable regularization scheme for a particular choice of $f_{\sigma}(k)$ that ensures that all the conditions above are satisfied. We identify the right-hand side of (5.13) with a finite assignment to the divergent series $\sum_{k=n}^{\infty} f_{\sigma}(k)$ in the generalized Euler-Maclaurin scheme. 
If the terms $S_{m}(n)\left[f_{\sigma}\right]$ in $\Gamma_{m}^{n}\left[f_{\sigma}\right]$ do not exist as $\sigma \rightarrow 0^{+}$, a finite assignment to $\sum_{k=n}^{\infty} f_{\sigma}(k)$ cannot be given in the limit. However, for such sums, one may be able to write

$$
\sum_{k=n}^{N} f_{\sigma}(k)=\sum_{k=n}^{n_{0}-1} f_{\sigma}(k)+\sum_{k=n_{0}}^{N} f_{\sigma}(k)
$$

for some $N>n_{0}>n \geq 0$ such that $S_{m}\left(n_{0}\right)\left[f_{\sigma}\right]$ exists, so that the sum $\sum_{k=n_{0}}^{\infty} f_{\sigma}(k)$ can be regularized. Then from (5.13) in the $N \rightarrow \infty$ limit:

$$
\lim _{\sigma \rightarrow 0^{+}}\left(\sum_{k=n_{0}}^{\infty} f_{\sigma}(k)-\mathcal{P}_{M}^{n_{0}}\left[f_{\sigma}\right]\right)=T_{M}^{n_{0}}+\beta_{M}^{n_{0}} .
$$

In terms of $n$ and $n_{0}$, we obtain the result

$$
\lim _{\sigma \rightarrow 0^{+}}\left(\sum_{k=n}^{\infty} f_{\sigma}(k)-\widehat{\mathcal{P}}_{M}^{n, n_{0}}\left[f_{\sigma}\right]\right)=T_{M}^{n_{0}}+\widehat{\beta}_{M}^{n, n_{0}}
$$

where

$$
\widehat{\Gamma}_{M}^{n, n_{0}}\left[f_{\sigma}\right] \equiv \sum_{k=n}^{n_{0}-1} f_{\sigma}(k)+\Gamma_{M}^{n_{0}}\left[f_{\sigma}\right]
$$

admits the series representation

$$
\widehat{\Gamma}_{M}^{n, n_{0}}\left[f_{\sigma}\right]=\widehat{\mathcal{P}}_{M}^{n, n_{0}}\left[f_{\sigma}\right]+\sum_{j=0}^{\infty} \widehat{\beta}_{M, j}^{n, n_{0}}\left[f_{\sigma}\right] \sigma^{j}
$$

with $\widehat{\mathcal{P}}_{M}^{n, n_{0}}\left[f_{\sigma}\right]$ containing only terms that are singular functions of $\sigma$ and

$$
\widehat{\beta}_{M}^{n, n_{0}} \equiv \lim _{\sigma \rightarrow 0^{+}} \sum_{j=0}^{\infty} \widehat{\beta}_{M, j}^{n, n_{0}}\left[f_{\sigma}\right] \sigma^{j}=\widehat{\beta}_{M, 0}^{n, n_{0}}\left[f_{\sigma}\right] .
$$

In summary, the assignment of a finite value $T_{M}^{n_{0}}+\widehat{\beta}_{M}^{n, n_{0}}$ to the divergent summation $\lim _{\sigma \rightarrow 0^{+}} \sum_{k=n}^{\infty} f_{\sigma}(k)$ is modeled on the process used by Hardy in [20] to assign a finite value to summations of the form $\sum_{k=n}^{\infty} f(k)$ using the Euler-Maclaurin identity.

We argue below that the sums $\lim _{q(\sigma) \rightarrow 0^{+}} \sum_{k=n}^{\infty} f_{q(\sigma)}(k)$ admit a viable regularization if they can be given the same finite assignment using this generalized summation for all real positive monotonic functions $q$ with $q(0)=0$. The assignment, when it exists, is computed by isolating terms in the Euler-Maclaurin identity that diverge as $q(\sigma) \rightarrow 0^{+}$and can be estimated with computable error bounds. In the following sections, this procedure is used to recover by analytic means standard results for a regularized energy and stress in a homogeneous dielectric, as well as a regularized energy in the smooth inhomogeneous dielectric contained in the open guide with only evanescent modes. In each case, the compensating singular terms are made explicit. 
For the three-dimensional Casimir problems under consideration here one is confronted with infinite range summations of the type (4.3) indexed by a triple of discrete indices with summands depending on the parameter $\sigma$. In the following, we exploit an approximation where $L_{x}$ and $L_{y}$ become large relative to a length scale $a \ll L_{x}, L_{y}$ in order to reduce the summation over $n_{x}, n_{y}$ to a parameterized single integral indexed by an integer so that the summation formula (5.16) becomes directly applicable.

\section{Quantum Induced Internal Energy and Stress in a Homogeneous Polarizable Medium}

The expectation value of the ground state energy due to a finite number $\underline{N}_{\max }$ of electromagnetic modes of type $s$ in the perfectly conducting cuboid cavity of dimensions $L_{x} \times L_{y} \times a$, filled with a homogenous (non-dispersive) medium of constant relative permittivity $\kappa_{0}>0$, is:

$$
\left\langle\widehat{H}^{s}\right\rangle=\frac{\hbar}{2} \sum_{\underline{\underline{\mathcal{N}}}}^{\underline{N}_{\max }} \omega_{\underline{\underline{\mathcal{N}}}}^{s}=\frac{\hbar c_{0}}{2 \sqrt{\kappa_{0}} a} \sum_{n_{x}}^{N_{x}} \sum_{n_{y}}^{N_{y}} \sum_{n_{z}}^{N_{z}} \Omega_{\underline{\underline{\mathcal{N}}}}^{s}
$$

where $\kappa_{0}\left(\omega_{\underline{\mathcal{N}}}^{T M} / c_{0}\right)^{2}=\kappa_{0}\left(\omega_{\underline{\mathcal{N}}}^{T E} / c_{0}\right)^{2}=k_{x}^{2}+k_{y}^{2}+k_{z}^{2}$ with $k_{x}=n_{x} \pi / L_{x}, k_{y}=n_{y} \pi / L_{y}$, $k_{z}=n_{z} \pi / a$ and $\underline{\mathcal{N}}=\left\{n_{x}, n_{y}, n_{z}\right\}$. For a cuboid cavity with $L_{x}, L_{y} \gg a$, the properties of the spectra as given by Theorem 4.1 — particularly the mode expansions (4.8) and (4.9) - enable one to reduce the triple sum (6.1) to a single summation over $n_{z}$. This is a particular consequence of the following theorem:

Theorem 6.1. For both the TE and TM modes with spectra defined in Theorem 4.1 for the perfectly conducting cuboid cavity filled with a homogeneous medium, when $L_{x}, L_{y} \gg$ a and $\underline{N}_{\max } \rightarrow \infty:$

$$
\lim _{\substack{L_{x} \rightarrow \infty \\ L_{y} \rightarrow \infty}} \frac{1}{L_{x} L_{y}} \sum_{\underline{\mathcal{N}}}^{\infty} \mathcal{J}_{\mathcal{M}}\left(k_{x}^{2}+k_{y}^{2}, n_{z}\right)=\frac{1}{2 \pi} \sum_{n_{z}=0}^{\infty} \int_{0}^{\infty} \mathcal{J}_{\mathcal{M}}\left(\rho^{2}, n_{z}\right) \rho d \rho
$$

where $\mathcal{J}_{\mathcal{M}}: \mathbb{R}^{+} \times \mathbb{R}^{+} \rightarrow \mathbb{R}$, provided the integrals exist. The label $\mathcal{M}$ is used below to distinguish different ground state expectation values.

Proof. When $L_{x}, L_{y} \gg a$ and $\underline{N}_{\max } \rightarrow \infty,\left\{k_{x}, k_{y}\right\}$ tend to a continuum with density of states measure $d k_{x} d k_{y}=\left(\pi^{2} / L_{x} L_{y}\right) d n_{x} d n_{y}$. For the $T E$ modes one has, after integrating over all directions of the transverse vector with components $\left(k_{x}, k_{y}\right)$ and $k_{x}, k_{y} \geq 0$, for any expression $\mathcal{J}_{\mathcal{M}}\left(k_{x}^{2}+k_{y}^{2}, n_{z}\right)$ :

$$
\begin{aligned}
\sum_{n_{z}=1}^{\infty} \sum_{n_{x}=1}^{\infty} \sum_{n_{y}=1}^{\infty} \mathcal{J}_{\mathcal{M}}\left(k_{x}^{2}+k_{y}^{2}, n_{z}\right) & \rightarrow \frac{L_{x} L_{y}}{2 \pi} \sum_{n_{z}=1}^{\infty} \int_{0}^{\infty} \mathcal{J}_{\mathcal{M}}\left(\rho^{2}, n_{z}\right) \rho d \rho \\
& \sum_{n_{z}=1}^{\infty} \sum_{n_{x}=1}^{\infty} \mathcal{J}_{\mathcal{M}}\left(k_{x}^{2}, n_{z}\right) \rightarrow \frac{L_{x}}{\pi} \sum_{n_{z}=1}^{\infty} \int_{0}^{\infty} \mathcal{J}_{\mathcal{M}}\left(k_{x}^{2}, n_{z}\right) d k_{x}
\end{aligned}
$$




$$
\sum_{n_{z}=1}^{\infty} \sum_{n_{y}=1}^{\infty} \mathcal{J}_{\mathcal{M}}\left(k_{y}^{2}, n_{z}\right) \rightarrow \frac{L_{y}}{\pi} \sum_{n_{z}=1}^{\infty} \int_{0}^{\infty} \mathcal{J}_{\mathcal{M}}\left(k_{y}^{2}, n_{z}\right) d k_{y}
$$

since in the large $L_{x}, L_{y}$ limit, the discrete transverse contribution $\rho^{2} \equiv k_{x}^{2}+k_{y}^{2}$ to the spectrum $\Omega$ tends to a continuum starting at $\rho=0$. Thus

$$
\begin{aligned}
\frac{1}{L_{x} L_{y}} \sum_{\underline{\underline{N}}}^{\infty} f\left(k_{x}^{2}+k_{y}^{2}, n_{z}\right) \rightarrow & \frac{1}{2 \pi} \sum_{n_{z}=1}^{\infty}\left(\int_{0}^{\infty} \mathcal{J}_{\mathcal{M}}\left(\rho^{2}, n_{z}\right) \rho d \rho\right. \\
& \left.+\frac{2}{L_{y}} \int_{0}^{\infty} \mathcal{J}_{\mathcal{M}}\left(k_{x}^{2}, n_{z}\right) d k_{x}+\frac{2}{L_{x}} \int_{0}^{\infty} \mathcal{J}_{\mathcal{M}}\left(k_{y}^{2}, n_{z}\right) d k_{y}\right)
\end{aligned}
$$

yielding

$$
\lim _{\substack{L_{x} \rightarrow \infty \\ L_{y} \rightarrow \infty}} \frac{1}{L_{x} L_{y}} \sum_{\underline{\mathcal{N}}}^{\infty} \mathcal{J}_{\mathcal{M}}\left(k_{x}^{2}+k_{y}^{2}, n_{z}\right)=\frac{1}{2 \pi} \sum_{n_{z}=1}^{\infty} \int_{0}^{\infty} \mathcal{J}_{\mathcal{M}}\left(\rho^{2}, n_{z}\right) \rho d \rho .
$$

The regularized contributions from the $T M$ modes follow a similar prescription to those of the TE modes. Specifically, when $L_{x}, L_{y} \gg a$ and $\underline{N}_{\max } \rightarrow \infty$ one has for any expression $\mathcal{J}_{\mathcal{M}}\left(k_{x}^{2}+k_{y}^{2}, n_{z}\right)$ :

$$
\sum_{n_{z}=0}^{\infty} \sum_{n_{x}=1}^{\infty} \sum_{n_{x}=1}^{\infty} \mathcal{J}_{\mathcal{M}}\left(k_{x}^{2}+k_{y}^{2}, n_{z}\right) \rightarrow \frac{L_{x} L_{y}}{2 \pi} \sum_{n_{z}=0}^{\infty} \int_{0}^{\infty} \mathcal{J}_{\mathcal{M}}\left(\rho^{2}, n_{z}\right) \rho d \rho
$$

so

$$
\lim _{\substack{L_{x} \rightarrow \infty \\ L_{y} \rightarrow \infty}} \frac{1}{L_{x} L_{y}} \sum_{\underline{\mathcal{N}}}^{\infty} \mathcal{J}_{\mathcal{M}}\left(k_{x}^{2}+k_{y}^{2}, n_{z}\right)=\frac{1}{2 \pi} \sum_{n_{z}=0}^{\infty} \int_{0}^{\infty} \mathcal{J}_{\mathcal{M}}\left(\rho^{2}, n_{z}\right) \rho d \rho
$$

and the result follows.

Since the regularization scheme developed in Sec.5 is purely mathematical, given a sequence of partial sums containing summands $f(k)$ there is no unique prescription to define $f_{\sigma}(k)$ compatible with the necessary properties leading to (5.13). In order to regularize sums involving expressions for ground state expectation values $\left\langle\mathcal{U}_{\mathcal{M}}\right\rangle$, we choose

$$
\mathcal{J}_{\mathcal{M}}\left(\rho^{2}, n_{z}\right) \equiv\left\langle\mathcal{U}_{\mathcal{M}}\right\rangle W_{q\left(\sigma^{\prime}\right)}\left(\rho^{2}, n_{z}\right)
$$

for some positive monotonic real-valued function $q$ of a dimensionless parameter $\sigma^{\prime} \in[0, \infty]$ with $q(0)=0$ and positive real-valued function $W_{q\left(\sigma^{\prime}\right)}: \mathbb{R}^{+} \times \mathbb{Z}^{+} \rightarrow \mathbb{R}$ with $\lim _{n_{z} \rightarrow 0} W_{q\left(\sigma^{\prime}\right)}\left(\rho^{2}, n_{z}\right)=0$ for all $\rho$. For physical applications the constant $\hbar$ used in the normalization conditions (4.2) is to be identified with the experimentally determined value of Planck's constant. This requirement is consistent with the demand that $W_{q(0)}\left(\rho^{2}, n_{z}\right)=1$ for all $q$. Since the mode normalizations must be maintained for all observables constructed in terms of electromagnetic fields this condition is required for the regularization of all auxiliary functions constructed 
from such $\mathcal{J}_{\mathcal{M}}\left(\rho^{2}, n_{z}\right)$. Thus from (6.1), writing $\sigma=q\left(\sigma^{\prime}\right)$ and choosing the dimensionless regulator $W_{\sigma}\left(\Omega_{\underline{\mathcal{N}}}^{T E}\right)=\exp \left(-\sigma \Omega_{\underline{\mathcal{N}}}^{T E}\right)$ to define

$$
\left\langle\widehat{H}^{T E}\right\rangle(\sigma) \equiv \frac{\hbar c_{0}}{2 \sqrt{\kappa_{0}} a} \sum_{n_{x}, n_{y}, n_{z}}^{\infty} \Omega_{\underline{\mathcal{N}}}^{T E} \exp \left(-\sigma \Omega_{\underline{\mathcal{N}}}^{T E}\right),
$$

one has for large $L_{x}, L_{y}$ compared with $a$, using (6.4):

$$
\begin{aligned}
\frac{\left\langle\widehat{H}^{T E}\right\rangle(\sigma)}{L_{x} L_{y}} & =\frac{\hbar c_{0}}{4 \sqrt{\kappa_{0}} a} \sum_{n_{z}=1}^{\infty} \int_{0}^{\infty} \rho \sqrt{\frac{\rho^{2} a^{2}}{\pi^{2}}+n_{z}^{2}} \exp \left(-\sigma \pi \sqrt{\rho^{2} a^{2} / \pi^{2}+n_{z}^{2}}\right) d \rho \\
& =\frac{\hbar c_{0} \pi^{2}}{8 \sqrt{\kappa_{0}} a^{3}} \sum_{n_{z}=1}^{\infty} \int_{n_{z}^{2}}^{\infty} \sqrt{u} \exp (-\sigma \pi \sqrt{u}) d u \\
& \equiv \frac{\hbar c_{0} \pi^{2}}{8 \sqrt{\kappa_{0}} a^{3}} \sum_{n_{z}=1}^{\infty} F_{\sigma}^{T E}\left(n_{z}\right)
\end{aligned}
$$

with $u\left(\rho^{2}, n_{z}\right)=\rho^{2} a^{2} / \pi^{2}+n_{z}^{2}$ and the dimensionless auxiliary function

$$
F_{\sigma}^{T E}\left(n_{z}\right)=\int_{n_{z}^{2}}^{\infty} \sqrt{u} \exp (-\sigma \pi \sqrt{u}) d u .
$$

For $M=2$, the error term $\lim _{\sigma \rightarrow 0^{+}} \varepsilon_{2}^{1}\left[F_{\sigma}^{T E}\right]=0$ and (5.7) yields the Laurent series expansion (see Appendix B.1):

$$
\Gamma_{2}^{1}\left[F_{\sigma}^{T E}\right]=\frac{12}{\pi^{4} \sigma^{4}}-\frac{2}{\pi^{3} \sigma^{3}}-\frac{1}{180}+\frac{\pi^{3} \sigma^{3}}{756}+O\left(\sigma^{4}\right) .
$$

From (5.8) and (5.13), this gives

$$
\lim _{\sigma \rightarrow 0^{+}}\left(\sum_{n_{z}=0}^{\infty} F_{\sigma}^{T E}\left(n_{z}\right)-\frac{12}{\pi^{4} \sigma^{4}}+\frac{2}{\pi^{3} \sigma^{3}}\right)=-\frac{1}{180}
$$

yielding a finite regularized expectation value for the $T E$ energy per unit area:

$$
\frac{\left\langle\widehat{H}^{T E}\right\rangle_{\mathrm{R}}}{L_{x} L_{y}} \equiv-\frac{\hbar c_{0} \pi^{2}}{1440 \sqrt{\kappa_{0}} a^{3}} .
$$

Similarly, using the dimensionless regulator $W_{\sigma}\left(\Omega_{\underline{\mathcal{N}}}^{T M}\right)=\exp \left(-\sigma \Omega_{\underline{\mathcal{N}}}^{T M}\right)$ to define

$$
\left\langle\widehat{H}^{T M}\right\rangle(\sigma) \equiv \frac{\hbar c_{0}}{2 \sqrt{\kappa_{0}} a} \sum_{n_{x}, n_{y}, n_{z}}^{\infty} \Omega_{\underline{\mathcal{N}}}^{T M} \exp \left(-\sigma \Omega_{\underline{\mathcal{N}}}^{T M}\right),
$$

one has for large $L_{x}, L_{y}$ using (6.5):

$$
\frac{\left\langle\widehat{H}^{T M}\right\rangle(\sigma)}{L_{x} L_{y}}=\frac{\hbar c_{0} \pi^{2}}{8 \sqrt{\kappa_{0}} a^{3}} \sum_{n_{z}=0}^{\infty} F_{\sigma}^{T M}\left(n_{z}\right)
$$


with $u\left(\rho^{2}, n_{z}\right)=\rho^{2} a^{2} / \pi^{2}+n_{z}^{2}$ and the dimensionless auxiliary function

$$
F_{\sigma}^{T M}\left(n_{z}\right)=\int_{n_{z}^{2}}^{\infty} \sqrt{u} \exp (-\sigma \pi \sqrt{u}) d u .
$$

This has the same structure as the dimensionless auxiliary function used to sum the $T E$ modes, but here the sum starts at $n_{z}=0$. For $M=2$, the error term $\lim _{\sigma \rightarrow 0^{+}} \varepsilon_{2}^{0}\left[F_{\sigma}^{T M}\right]=0$ and (5.7) yields the Laurent series expansion (see Appendix B.1):

$$
\Gamma_{2}^{0}\left[F_{\sigma}^{T M}\right]=\frac{12}{\pi^{4} \sigma^{4}}+\frac{2}{\pi^{3} \sigma^{3}}-\frac{1}{180} .
$$

From (5.8) and (5.13), this gives

$$
\lim _{\sigma \rightarrow 0^{+}}\left(\sum_{n_{z}=0}^{\infty} F_{\sigma}^{T M}\left(n_{z}\right)-\frac{12}{\pi^{4} \sigma^{4}}-\frac{2}{\pi^{3} \sigma^{3}}\right)=-\frac{1}{180}
$$

yielding a finite regularized expectation value for the $T M$ energy per unit area:

$$
\frac{\left\langle\widehat{H}^{T M}\right\rangle_{\mathrm{R}}}{L_{x} L_{y}}=-\frac{\hbar c_{0} \pi^{2}}{1440 \sqrt{\kappa_{0}} a^{3}},
$$

which coincides with (6.8). Therefore the ground state expectation value of the total energy per unit area is:

$$
\frac{\langle\widehat{H}\rangle_{\mathrm{R}}}{L_{x} L_{y}}=\frac{\left\langle\widehat{H}^{T E}\right\rangle_{\mathrm{R}}}{L_{x} L_{y}}+\frac{\left\langle\widehat{H}^{T M}\right\rangle_{\mathrm{R}}}{L_{x} L_{y}}=-\frac{\hbar c_{0} \pi^{2}}{720 \sqrt{\kappa_{0}} a^{3}}
$$

which reduces to Casimir's result [1] when $\kappa_{0}=1$.

It is straightforward to show that for any $p>0$, a regulator of the form $W_{\sigma}\left(\Omega_{\mathcal{N}_{s}}^{s}\right)=\exp \left(-\sigma\left(\Omega_{\mathcal{N}_{s}}^{s}\right)^{p}\right)$ will yield the same finite regularized energy expectation value per unit area independent of $p$ for both the TE and TM modes. However, the exposed singular behavior in $\sigma$ is $p$-dependent. Although this falls short of a rigorous proof, it lends credence to our argument that this Euler-Maclaurin regularization scheme is independent of the regulator involved.

A calculation of the regularized ground state expectation value of the electromagnetic stress tensor in the dielectric requires the complete set of system eigenmode functions. This has been obtained in Sec. 3 for the cuboid containing a homogeneous medium and the smooth inhomogeneous medium with relative permittivity (3.10). ${ }^{\mathrm{c}}$

To extract a finite stress expectation at any point labeled by $(x, y, z)$ in a dielectric with electromagnetic mode spectra labeled by the integers $\left\{n_{x}, n_{y}, \ell\right\}$ define, for all $i, j$, the auxiliary functions

$$
\mathcal{S}_{i j}^{s}(\sigma, x, y, z)=\sum_{\ell}^{\infty} F_{\sigma, i j}^{s}(\ell, x, y, z)
$$

'This information enables one to construct the appropriate electromagnetic Green's function for each system. 
where, from (4.12), for some regulator $W_{\sigma}$ :

$$
F_{\sigma, i j}^{s}(\ell, x, y, z)=\sum_{n_{x}}^{\infty} \sum_{n_{y}}^{\infty}\left\langle\left(\widehat{S}_{\mathcal{N}_{s}}^{s}\right)_{i j}\right\rangle(x, y, z) W_{\sigma}\left(\Omega_{n_{x}, n_{y}, \ell}^{s}\right) .
$$

In principle one could now apply (5.13) to $F_{\sigma, i j}^{s}(\ell, x, y, z)$ for fixed $\sigma, x, y, z$ and all $i, j$. Analyzing $\Gamma_{M}^{n}\left[F_{\sigma, i j}^{s}\right]$ or $\widehat{\Gamma}_{M}^{n, n_{0}}\left[F_{\sigma, i j}^{s}\right]$ as a function of $\sigma$ in order to extract a regularized local stress in the dielectric would undoubtedly be computationally intensive. However using this approach to calculate the total normal force acting on either side of any cross-section where $z$ is the constant $z_{0}$ is less involved. The contribution from type $s$ modes to such a regularized force is generated from

$$
\mathcal{F}^{s}\left(\sigma, z_{0}\right)=\sum_{\ell}^{\infty} \bar{F}_{\sigma}^{s}\left(\ell, z_{0}\right)
$$

where

$$
\bar{F}_{\sigma}^{s}\left(\ell, z_{0}\right)=\sum_{n_{x}}^{\infty} \sum_{n_{y}}^{\infty} \int_{0}^{L_{x}} \int_{0}^{L_{y}}\left\langle\left(\widehat{S}_{\mathcal{N}_{s}}^{s}\right)_{33}\right\rangle\left(x, y, z_{0}\right) W_{\sigma}\left(\Omega_{n_{x}, n_{y}, \ell}^{s}\right) d x d y .
$$

After the integration over $x$ and $y$ the $n_{x}, n_{y}$ dependence of the summands in $\bar{F}_{\sigma}^{s}\left(\ell, z_{0}\right)$ arises from only $k_{x}^{2}+k_{y}^{2}$. Hence if $L_{x}, L_{y}$ are large compared with the inhomogeneity scale parameter $a$ in (3.10) it follows from (6.4) and (6.5) that

$$
\bar{F}_{\sigma}^{s}\left(\ell, z_{0}\right)=\frac{L_{x} L_{y}}{2 \pi} \int_{0}^{\infty} \rho \bar{f}^{s}\left(\ell, z_{0}, \rho\right) \widetilde{W}_{\sigma}^{s}(\ell, \rho) d \rho
$$

where $\widetilde{W}_{\sigma}^{s}(\ell, \rho)=W_{\sigma}\left(\Omega_{n_{x}, n_{y}, \ell}^{s}\right)$ and

$$
\bar{f}^{s}\left(\ell, z_{0}, \sqrt{k_{x}^{2}+k_{y}^{2}}\right)=\int_{0}^{L_{x}} \int_{0}^{L_{y}}\left\langle\left(\widehat{S}_{n_{x}, n_{y}, \ell}\right)_{33}\right\rangle\left(x, y, z_{0}\right) d x d y .
$$

Thus the triple indexed summation over $\left\{n_{x}, n_{y}, \ell\right\}$ reduces to a single infinite range summation (over $\ell$ ) with single integrals (over $\rho$ ) as summands. Equation (5.13) or (5.17) can then be used to generate $T_{M}^{n}+\beta_{M}^{n}$ or $T_{M}^{n_{0}}+\widehat{\beta}_{M}^{n, n_{0}}$ respectively for both $s \in\{T E, T M\}$. To implement this regularization scheme analytically and extract a finite "renormalized" value for the total expectation value of the normal average Casimir pressure in the dielectric ${ }^{\mathrm{d}}$ on either side of any plane $z=z_{0}$, after dividing by the area $L_{x} L_{y}$, an explicit formula for each auxiliary function is required.

The regularized ground state expectation value of the normal force $\langle\widetilde{\mathcal{F}}\rangle_{\mathrm{R}}$ at the boundary $z=z_{0}$ of the cuboid cavity with constant $\kappa_{1}=\kappa_{0}$ can be extracted

\footnotetext{
${ }^{\mathrm{d}}$ One may verify that this normal pressure is the total pressure since, at any fixed plane $z=z_{0}$, the tangential forces are zero. Regularized pressures are, of course, equal in magnitude but act in opposite directions on such planes with opposite normals.
} 
using (6.13) and (6.12) with $\ell=n_{z}$ and the dimensionless regulator $W_{\sigma}\left(\Omega_{\mathcal{N}_{s}}^{s}\right)=$ $\exp \left(-\sigma \Omega_{\mathcal{N}_{s}}^{s}\right)$. Define

$$
\frac{\mathcal{F}^{T E}\left(\sigma, z_{0}\right)}{L_{x} L_{y}}=\frac{\hbar c_{0} \pi^{2}}{8 \sqrt{\kappa_{0}} a^{4}} \sum_{n_{z}=1}^{\infty} \bar{F}_{\sigma}^{T E}\left(n_{z}\right)
$$

in terms of the dimensionless auxiliary function

$$
\bar{F}_{\sigma}^{T E}\left(n_{z}\right)=\int_{n_{z}^{2}}^{\infty} \frac{n_{z}^{2}}{\sqrt{u}} \exp (-\sigma \pi \sqrt{u}) d u .
$$

With $M=2$, the error term $\lim _{\sigma \rightarrow 0^{+}} \varepsilon_{2}^{1}\left[\bar{F}_{\sigma}^{T E}\right]=0$ and (5.7) gives the Laurent series expansion (see Appendix B.2)

$$
\Gamma_{2}^{1}\left[\bar{F}_{\sigma}^{T E}\right]=\frac{4}{\pi^{4} \sigma^{4}}-\frac{1}{60}+\frac{\pi^{3} \sigma^{3}}{504}+O\left(\sigma^{4}\right)
$$

with (5.8) yielding

$$
\lim _{\sigma \rightarrow 0^{+}}\left(\sum_{n_{z}=1}^{\infty} \bar{F}_{\sigma}^{T E}\left(n_{z}\right)-\frac{4}{\pi^{4} \sigma^{4}}\right)=-\frac{1}{60} .
$$

Hence the finite $T E$ regularized contribution to the pressure is:

$$
\frac{\left\langle\mathcal{F}^{T E}\left(z_{0}\right)\right\rangle_{\mathrm{R}}}{L_{x} L_{y}}=-\frac{\hbar c_{0} \pi^{2}}{480 \sqrt{\kappa_{0}} a^{4}} .
$$

For the $T M$ modes

$$
\frac{\mathcal{F}^{T M}\left(\sigma, z_{0}\right)}{L_{x} L_{y}}=\frac{\hbar c_{0} \pi^{2}}{8 \sqrt{\kappa_{0}} a^{4}} \sum_{n_{z}=0}^{\infty} \bar{F}_{\sigma}^{T M}\left(n_{z}\right)
$$

in terms of the dimensionless auxiliary function

$$
\bar{F}_{\sigma}^{T M}\left(n_{z}\right)=\int_{n_{z}^{2}}^{\infty} \frac{n_{z}^{2}}{\sqrt{u}} \exp (-\sigma \pi \sqrt{u}) d u
$$

which is the same as that for the TE modes. With $M=2$, the error term $\lim _{\sigma \rightarrow 0^{+}}$ $\varepsilon_{2}^{0}\left[\bar{F}_{\sigma}^{T M}\right]=0$ and (5.7) gives the Laurent series expansion (see Appendix B.2)

$$
\Gamma_{2}^{0}\left[\bar{F}_{\sigma}^{T M}\right]=\frac{4}{\pi^{4} \sigma^{4}}-\frac{1}{60}
$$

with (5.8) yielding

$$
\lim _{\sigma \rightarrow 0^{+}}\left(\sum_{n_{z}=1}^{\infty} \bar{F}_{\sigma}^{T M}\left(n_{z}\right)-\frac{4}{\pi^{4} \sigma^{4}}\right)=-\frac{1}{60} .
$$

Hence the finite $T M$ regularized contribution to the pressure is:

$$
\frac{\left\langle\mathcal{F}^{T M}\left(z_{0}\right)\right\rangle_{\mathrm{R}}}{L_{x} L_{y}}=-\frac{\hbar c_{0} \pi^{2}}{480 \sqrt{\kappa_{0}} a^{4}}
$$


which is an equal contribution to the pressure as that from the TE modes. Thus, the ground state expectation value of the total regularized (tensile) pressure on the plate at $z=z_{0}$ is then

$$
\frac{\left\langle\mathcal{F}\left(z_{0}\right)\right\rangle_{\mathrm{R}}}{L_{x} L_{y}}=\frac{\left\langle\mathcal{F}^{T E}\left(z_{0}\right)\right\rangle_{\mathrm{R}}}{L_{x} L_{y}}+\frac{\left\langle\mathcal{F}^{T M}\left(z_{0}\right)\right\rangle_{\mathrm{R}}}{L_{x} L_{y}}=-\frac{\hbar c_{0} \pi^{2}}{240 \sqrt{\kappa_{0}} a^{4}},
$$

which reduces to Casimir's result [1] when $\kappa_{0}=1$. Once again, any regulator of the form $W_{\sigma}\left(\Omega_{\mathcal{N}_{s}}^{s}\right)=\exp \left(-\sigma\left(\Omega_{\mathcal{N}_{s}}^{s}\right)^{p}\right)$ for $p>0$ produces the same regularized value. Thus for this system with a homogeneous medium between the planes, one has the expected result:

$$
\frac{\left\langle\mathcal{F}\left(z_{0}\right)\right\rangle_{\mathrm{R}}}{L_{x} L_{y}}=-\frac{\partial}{\partial a}\left(\frac{\langle\widehat{H}\rangle_{\mathrm{R}}}{L_{x} L_{y}}\right)
$$

\section{Quantum Induced Internal Energy and Stress in a Smooth Inhomogeneous Polarizable Medium}

The calculation of the ground state expectation value of the electromagnetic energy in the open waveguide containing the smooth inhomogeneous medium with permittivity given in (3.10) is based on the angular frequency mode spectrum given by

$$
\left(\omega_{n_{x}, n_{y}, \ell}^{s}\right)^{2}=\frac{c_{0}^{2}}{a^{2} \kappa_{0}}\left(\Omega_{n_{x}, n_{y}, \ell}^{s}\right)^{2}=\frac{c_{0}^{2}}{a^{2} \kappa_{0}}\left(\ell+\sqrt{\theta^{s}}\right)\left(\ell+\sqrt{\theta^{s}}+1\right)
$$

where the $n_{x}, n_{y}$ dependence of $\theta^{s}$ is given by (3.11) and $n_{x}, n_{y}, \ell=0,1,2, \ldots$ Following the previous strategy we have:

Theorem 7.1. With $L_{x}, L_{y} \gg$, the inhomogeneity scale in (3.10), the ground state energy expectation value per unit area in the guide can be determined from:

$$
\frac{\langle\widehat{H}\rangle(\sigma)}{L_{x} L_{y}}=\frac{\hbar c_{0}}{8 \pi \sqrt{\kappa_{0}} a^{3}}\left(\sum_{\ell=0}^{\infty} F_{\sigma}^{T E}(\ell)+\sum_{\ell=0}^{\infty} F_{\sigma}^{T M}(\ell)\right)
$$

where, using the dimensionless regulator $W_{\sigma}\left(\Omega_{n_{x}, n_{y}, \ell}^{\sigma}\right)=\exp \left(-\sigma \Omega_{n_{x}, n_{y}, \ell}^{s}\right)$, one has dimensionless auxiliary functions

$$
F_{\sigma}^{T E}(\ell)=\int_{\ell(\ell+1)}^{\infty} \mathcal{F}_{\ell}(u) \exp (-\sigma \sqrt{u}) d u
$$

and

$$
F_{\sigma}^{T M}(\ell)=\int_{(\ell+1)(\ell+2)}^{\infty} \mathcal{F}_{\ell}(u) \exp (-\sigma \sqrt{u}) d u
$$

with

$$
\mathcal{F}_{\ell}(u)=\sqrt{u}\left(1-\frac{1+2 \ell}{\sqrt{1+4 u}}\right)
$$


As before, for large $L_{x}, L_{y}$ compared with $a$, the summands in (7.1) have reduced to single quadratures since the medium inhomogeneity depends only on $z$. To apply (5.13) for the TE modes, it is necessary to start the summation on the left-hand side of (5.1) at $n=1$ in order to have well defined limits on the right-hand side as $N$ tends to infinity. It is shown in Appendix C that, with $M=3$ :

$$
\lim _{\sigma \rightarrow 0^{+}}\left(\sum_{\ell=0}^{\infty} F_{\sigma}^{T E}(\ell)-\frac{6}{\sigma^{4}}-\frac{1}{24 \sigma^{2}}+\frac{1}{384} \ln (\sigma)\right)=0.00393263 \pm 6.40 \times 10^{-6}
$$

and

$$
\lim _{\sigma \rightarrow 0^{+}}\left(\sum_{\ell=0}^{\infty} F_{\sigma}^{T M}(\ell)-\frac{6}{\sigma^{4}}+\frac{23}{24 \sigma^{2}}+\frac{49}{384} \ln (\sigma)\right)=0.07349016 \pm 3.17 \times 10^{-5}
$$

to 6 significant figures. Hence

$$
\begin{aligned}
& \lim _{\sigma \rightarrow 0^{+}}\left(\sum_{\ell=0}^{\infty} F_{\sigma}^{T E}(\ell)+\sum_{\ell=0}^{\infty} F_{\sigma}^{T M}(\ell)-\frac{12}{\sigma^{4}}+\frac{11}{12 \sigma^{2}}+\frac{25}{192} \ln (\sigma)\right) \\
& \quad=0.0774228 \pm 3.81 \times 10^{-5},
\end{aligned}
$$

to 6 significant figures, yielding a finite positive regularized quantum ground state energy per unit area:

$$
\frac{\langle\widehat{H}\rangle_{\mathrm{R}}}{L_{x} L_{y}}=0.07742 \frac{\hbar c_{0}}{8 \pi \sqrt{\kappa_{0}} a^{3}}=0.2247\left(\frac{\hbar c_{0} \pi^{2}}{720 \sqrt{\kappa_{0}} a^{3}}\right)
$$

in the smooth inhomogeneous dielectric, which is approximately one fifth of the magnitude of the regularized quantum ground state energy per unit area associated with the canonical Casimir parallel plate system (with gap a) filled with a homogeneous dielectric with relative permittivity $\kappa_{0}$ (6.11), to 4 significant figures.

In 29], a numerical algorithm based on the Abel-Plana formula (see Appendix D was developed in order to estimate numerically certain values assigned to sums that arose in estimating the quantum induced force difference between plates confining a different smooth inhomogeneous dielectric medium. Since in that problem one could not solve for the spectra analytically, it was assumed that mode sums could be regularized using an exponential regulator in a variable $s$ and the algorithm employed a novel filtering method to numerically fit the regularized sums to Laurent series in $s$ in order to extract the coefficients independent of $s$. In this article, we have denoted the regularizing parameter by $\sigma$ instead of $s$.

Motivated by the results derived above from an analysis of $\Gamma_{M}^{n}\left[f_{\sigma}\right]$ for various functions $f_{\sigma}$ based on the Euler-Maclaurin scheme, a natural generalization of the filtering algorithm developed in [29] is here proposed by including terms containing powers and logarithmic functions of $\sigma$ in the numerical fits and taking into account the criteria and error bounds implicit in its formulation. For example, if it is assumed that a sum of the form $\sum_{k=n}^{\infty} f_{\sigma}(k)$ is to be regularized one proceeds to numerically 
approximate $\Gamma(\sigma) \equiv \Gamma_{m}^{n}\left[f_{\sigma}\right]$ given by (5.7) for some $n$ and choice of positive integer parameters $m, J_{1}, J_{2}, K_{1}, K_{2}$ according to

$$
\Gamma(\sigma)=\sum_{j=-J_{1}}^{J_{2}} \sum_{k=-K_{1}}^{K_{2}} c_{j, k}(\ln (\sigma))^{j} \sigma^{k} \quad\left(c_{j, k} \in \mathbb{R}, \sigma>0\right)
$$

with $J_{2}>J_{1} \geq 0, K_{2}>K_{1} \geq 0$. The algorithm developed in [29] can be generalized to seek a stable optimal fit in that parameter domain for values of $m \geq M$. From such a fit, one may extract the value of $c_{0,0}$ yielding a numerical estimate of the associated regularized sum. In parallel, one may use the bounds (5.14) to calculate $\lim _{\sigma \rightarrow 0^{+}} \varepsilon_{m}^{n}\left[f_{\sigma}\right]$ as a function of $m$ and hence estimate the relative error that can be assigned to the optimal values of $c_{0,0}$.

However as noted in Sec. 5, in some cases the choice of $n$ may necessitate the isolation of certain terms in $\Gamma_{m}^{n}\left[f_{\sigma}\right]$ according to (5.15). This is achieved by choosing a value of some integer $n_{0}>n$ such that $S_{m}\left(n_{0}\right)\left[f_{\sigma}\right]$ has a finite value. In such cases, one proceeds as above but with $\Gamma(\sigma) \equiv \widehat{\Gamma}_{m}^{n, n_{0}}\left[f_{\sigma}\right]$ as given by (5.17).

In the current context one may test the efficacy of such types of ansätz with $f_{\sigma}=F_{\sigma}^{s}$ using (C.4) and (C.10) in Appendix C by applying the same algorithm to a fit of the simpler form:

$$
\Gamma^{s}(\sigma)=c_{L}^{s} \ln (\sigma)+\sum_{j=0}^{\mathfrak{N}} \frac{c_{j}^{s}}{\sigma^{j}} \quad\left(c_{L}^{s}, c_{j}^{s} \in \mathbb{R}\right),
$$

if one chooses a range of $\sigma$ suitably close to $\sigma=0$. It can be seen from Figs. 44 7 that with this ansätz the algorithm yields excellent agreement with the analytic results for both the $T E$ and $T M$ mode contributions to the regularized quantum

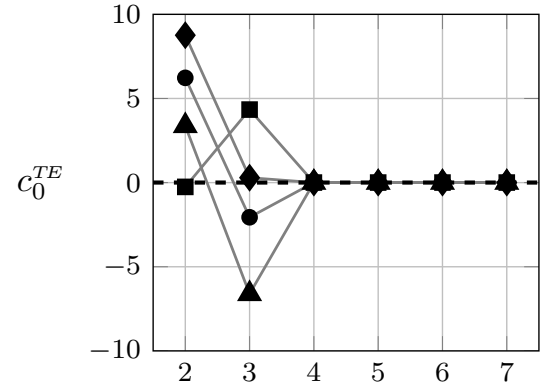

$\mathfrak{N}$

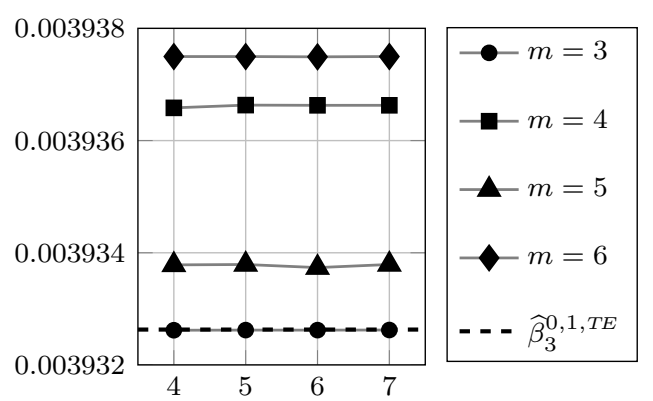

$\mathfrak{N}$

Fig. 4. Numerical values of the constant $c_{0}^{T E}$ in $(7.8)$ as a function of the largest inverse power $\mathfrak{N}$, derived from fitting this form to the values of $\widehat{\Gamma}_{m}^{0,1}\left[F_{\sigma}^{T E}\right]$ given by (5.7) over the range $1 / 20000 \leq$ $\sigma \leq 1 / 2000$ for the various values of $m$ indicated in the legend. The plot on the right indicates these variations of $c_{0}^{T E}$ on an expanded scale. The numerical values of $c_{0}^{T E}$ are compared with the value $\widehat{\beta}_{3}^{0,1, T E}$ determined as the $\sigma$-independent term in the analytic series representation of $\widehat{\Gamma}_{m}^{0,1}\left[F_{\sigma}^{T E}\right]$ in (5.18). 


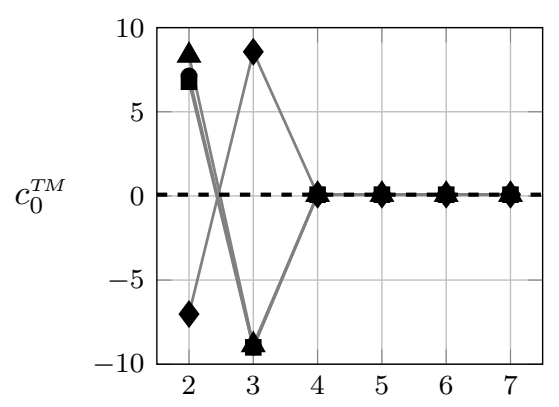

$\mathfrak{N}$

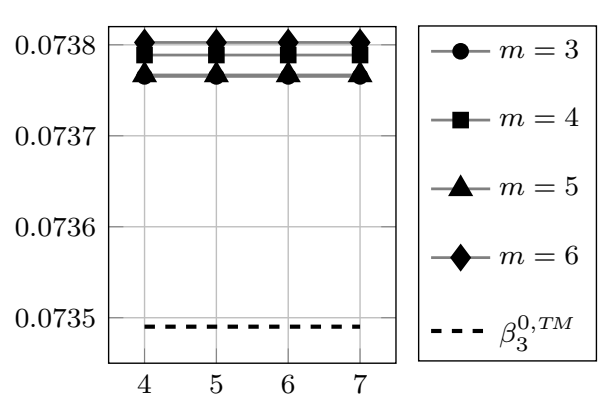

$\mathfrak{N}$

Fig. 5. Numerical values of the constant $c_{0}^{T M}$ in (7.8) as a function of the largest inverse power $\mathfrak{N}$, derived from fitting this form to the values of $\Gamma_{m}^{0}\left[F_{\sigma}^{T M}\right]$ given by (5.7) over the range $1 / 20000 \leq$ $\sigma \leq 1 / 2000$ for the various values of $m$ indicated in the legend. The plot on the right indicates these variations of $c_{0}^{T M}$ on an expanded scale. The numerical values of $c_{0}^{T M}$ are compared with the value $\beta_{3}^{0, T M}$ determined as the $\sigma$-independent term in the analytic series representation of $\Gamma_{3}^{0}\left[F_{\sigma}^{T M}\right]$ in $(5.8)$.
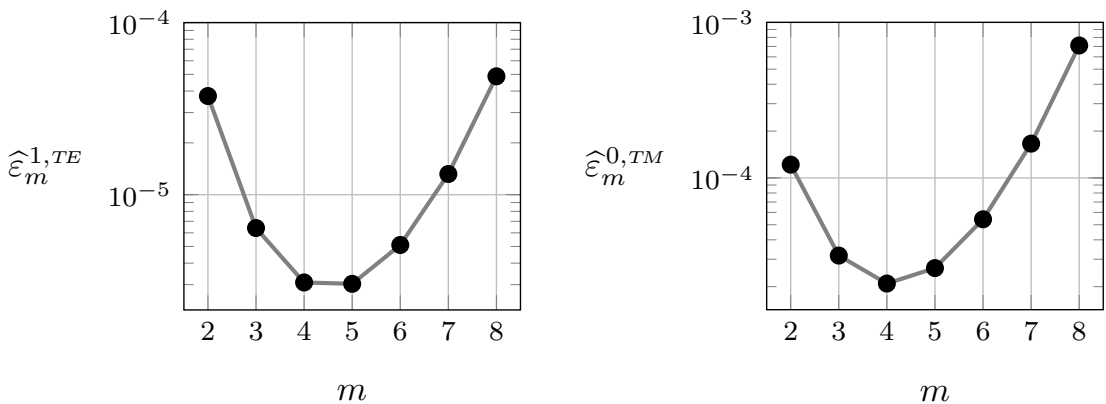

Fig. 6. Variation of $\widehat{\varepsilon}_{m}^{n_{s}, s} \equiv \lim _{\sigma \rightarrow 0}+\varepsilon_{m}^{n_{s}}\left[F_{\sigma}^{s}\right]$ with $m$ for contributions from the TE modes on the left and the $T M$ modes on the right, calculated numerically from (5.14). For each $s \in\{T E, T M\}$ and corresponding $n_{s} \in\{0,1\}$, a value of $m$ in the vicinity of a local minimum of these curves offers a potential value of $M_{s}$ and a corresponding relative error $\left|\widehat{\varepsilon}_{M_{s}}^{n_{s}, s} / \beta_{M_{s}}^{n_{s}, s}\right|$ for the numerical estimate of $\beta_{M_{s}}^{n_{s}, s}$ determined in Figs. 4 and 5

induced energy per unit area inside the ENZ type smooth inhomogeneous dielectric to 6 significant figures.

Given the evanescent behavior of the dielectric eigen-modes in the open guide as $|z|$ tends to infinity it is tempting to exploit expression (7.6) for the global energy per unit area to estimate the quantum pressure on a pair of perfectly conducting planes inserted in the location of the planes $z= \pm a / 2$. For $L_{x}, L_{y} \gg a$ this effectively transforms the open guide into a pair of parallel conducting plates separated by a gap of width $a$, containing a dielectric with permittivity approximating that given by (3.10). However there is no a priori reason to expect that differentiating (17.6) with respect to the parameter $a$ would give an estimate of the induced 


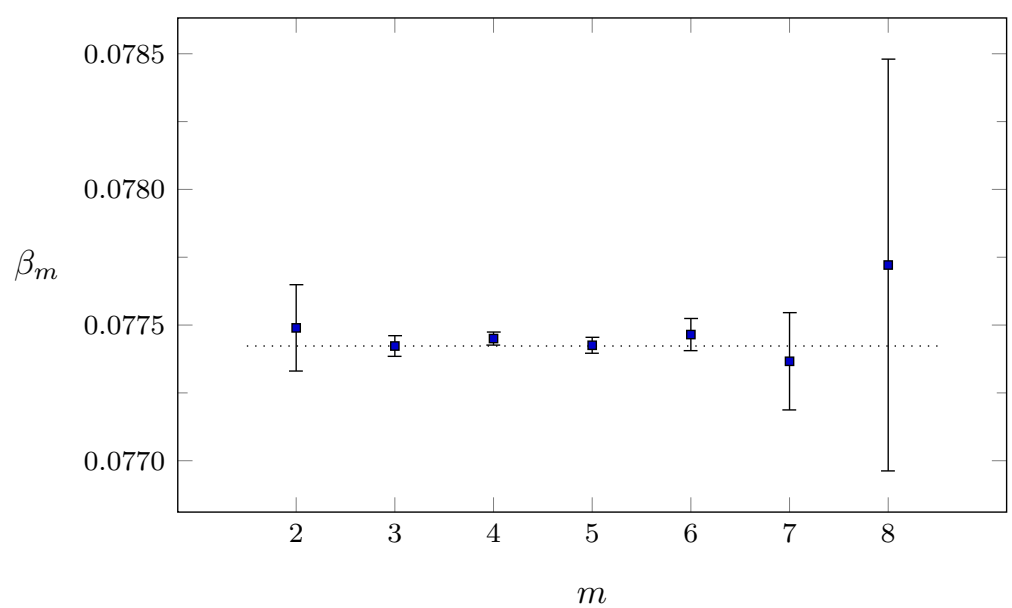

Fig. 7. This figure shows how the results of the numerical algorithm used to determine $\beta_{m} \equiv \widehat{\beta}_{m}^{0,1, T E}+\beta_{m}^{0, T M}$ compare favorably with the analytically calculated values indicated here in boldface for various $m$. Their associated error bounds are determined from $\lim _{\sigma \rightarrow 0^{+}}\left(\varepsilon_{m}^{1}\left[F_{\sigma}^{T E}\right]+\varepsilon_{m}^{0}\left[F_{\sigma}^{T M}\right]\right)$ by numerical integration of the integral in (5.14). The dotted line passes through the value of $c_{0}^{T E}+c_{0}^{T M}$ determined by the numerical algorithm above with $m=3$.

electromagnetic pressure at the inserted plates in the presence of the smooth inhomogeneous dielectric medium.

From the general formulation given in Sec. 6, the total normal force acting on either side of any cross-section at $z=z_{0}$ in the guide can be determined, for some $n, M$, in terms of $\Gamma_{M}^{n}\left[\bar{F}_{\sigma}^{s}\right]$ or $\widehat{\Gamma}_{M}^{n, n_{0}}\left[\bar{F}_{\sigma}^{s}\right]$ once the $\bar{F}_{\sigma}^{s}$ are known analytically from (6.13) and (6.14). The integration in (6.14) can be performed analytically for both $s=T E$ and $s=T M$ yielding

$$
\bar{f}^{T E}\left(\ell, z_{0}, \rho\right)=\frac{\hbar c_{0}^{2}}{4 \kappa_{0} \omega_{\underline{\mathcal{N}}}^{T E} a^{2} I_{\underline{\mathcal{N}}}^{T E}}\left[\left(\frac{d \mathcal{Z}_{\theta^{T E}, \ell}}{d Z}\right)^{2}-\mathcal{Z}_{\theta^{T E}, \ell} \frac{d^{2} \mathcal{Z}_{\theta^{T E}, \ell}}{d Z^{2}}\right]_{Z=z_{0} / a}
$$

and

$$
\begin{aligned}
\bar{f}^{T M}\left(\ell, z_{0}, \rho\right)= & \frac{\hbar c_{0}^{2}}{4 \kappa_{0} \omega_{\underline{\mathcal{N}}}^{T M} a^{2} I_{\underline{\mathcal{N}}}^{T M}}\left[\left(\frac{d \mathcal{Z}_{\theta^{T M}, \ell}}{d Z}\right)^{2}-\frac{\mathcal{Z}_{\theta^{T M}, \ell}}{\cosh (Z)} \frac{d^{2}}{d Z^{2}}\left(\cosh (Z) \mathcal{Z}_{\theta T M, \ell}\right)\right. \\
& \left.+\frac{\mathcal{Z}_{\theta^{T M}, \ell}^{2}}{\cosh ^{2}(Z)}\left(3 \cosh (Z)^{2}-1\right)\right]_{Z=z_{0} / a}
\end{aligned}
$$

with $\theta^{T E}=a^{2} \rho^{2}$ and $\theta^{T M}=a^{2} \rho^{2}+1$, though the computation of the integral (6.13) appears resistant to analytic evaluation. Despite this technical hurdle the regularization process based on the Euler-Maclaurin formula is directly applicable and since all integrals in the formula yield finite values, in the absence of a strictly analytic evaluation of (6.13) the numerical approach outlined above is available. Given a user defined tolerance one can seek suitable values for the number of terms 
(determined by $n, n_{0}$ and $M$ ) to include in the regularized assignment compatible with this tolerance. If such values exist one has a numerical estimate with a well defined error bound determined by $T_{M}^{n}$ or $T_{M}^{n_{0}}$ for the regularized average quantum stress across any cross-section of the smooth inhomogeneous dielectric in the open guide.

\section{Density of States in the Large Volume Continuum Limit}

Since the spectrum of electromagnetic states in the particular inhomogeneous ENZ dielectric considered in this article is known analytically it is of interest to calculate the asymptotic behavior of their density as a function of specific angular frequency $\Omega$ in the large volume limit:

Theorem 8.1. In the limit when $L_{x}, L_{y}$ become large with $L_{x} / a$ and $L_{y} / a$ finite, the continuum limit for the density of states for TE and TM modes in the open guide behaves like:

$$
\frac{d \mathcal{N}^{s}}{d \Omega} \simeq \frac{L^{2}}{4 \pi a^{2}}\left(\Omega^{2}-\Omega\right)
$$

Proof. Since the dependence of the spectral parameter $\Omega_{n_{x}, n_{y}, \ell}^{s}$ on the geometrical parameters is known explicitly one may calculate a density of states in the limit when $L_{x}, L_{y}$ become large with $L_{x} / a$ and $L_{y} / a$ finite by counting the number of eigen-modes $\mathcal{N}^{s}(\Omega)$ of type $s$ with spectral parameters less than or equal to some $\Omega$ in this limit 30 . As the geometrical parameters increase the discrete spectrum tends to a continuum that populates a finite volume

$$
\mathcal{N}^{s}(\Omega)=\left|\int_{\mathcal{V}_{\Omega}^{s}} d \ell \wedge d n_{x} \wedge d n_{y}\right|
$$

in the Cartesian space with coordinates $n_{x}, n_{y}, \ell$. When $L_{x}=L_{y}=L$ the compact domains $\mathcal{V}_{\Omega}^{s}$ are bounded by portions of the planes $\ell=0, n_{x}=0, n_{y}=0$ and the iso-spectral surfaces of revolution given implicitly by:

$$
\Omega^{2}-\left(\ell+\frac{a \pi}{L} \sqrt{n_{x}^{2}+n_{y}^{2}}\right)\left(\ell+\frac{a \pi}{L} \sqrt{n_{x}^{2}+n_{y}^{2}}+1\right)=0
$$

for the $T E$ modes (for these modes $\mathcal{V}_{\Omega}^{T E}$ is a quarter of the volume of a right circular cone based on the plane $\ell=0$ ) and

$$
\Omega^{2}-\left(\ell+\sqrt{\frac{a^{2} \pi^{2} n_{x}^{2}}{L^{2}}+\frac{a^{2} \pi^{2} n_{y}^{2}}{L^{2}}+1}\right)\left(\ell+\sqrt{\frac{a^{2} \pi^{2} n_{x}^{2}}{L^{2}}+\frac{a^{2} \pi^{2} n_{y}^{2}}{L^{2}}+1}+1\right)=0
$$

for the $T M$ modes. These surfaces can be conveniently parameterized as:

$$
\ell=\frac{1}{2} \sqrt{4 \Omega^{2}+1}-\frac{1}{2}-\mathcal{F}^{s}(u), \quad n_{x}=\frac{L}{a \pi} u \cos \phi, \quad n_{y}=\frac{L}{a \pi} u \sin \phi
$$

with $\mathcal{F}^{T E}(u)=u, \mathcal{F}^{T M}(u)=\sqrt{u^{2}+1}$ and

$$
0 \leq u \leq u_{0}^{s}, \quad 0 \leq \phi \leq \frac{\pi}{2}
$$


where $u_{0}^{s}$ is the positive root of $\ell\left(u_{0}^{s}\right)=0$, yielding

$$
\mathcal{V}_{\Omega}^{s}=\left(\frac{L}{\pi a}\right)^{2} \cdot \frac{\pi}{2} \cdot \int_{0}^{u_{0}^{s}} \ell(u) u d u
$$

with

$$
u_{0}^{T E}=\frac{1}{2} \sqrt{4 \Omega^{2}+1}-\frac{1}{2} \quad \text { and } \quad u_{0}^{T M}=\frac{1}{2} \sqrt{4 \Omega^{2}-2 \xi-2}
$$

where $\xi=\sqrt{4 \Omega^{2}+1}$. Then

$$
\begin{aligned}
\mathcal{V}_{\Omega}^{T E} & =\frac{L^{2}}{96 \pi a^{2}}\left(\sqrt{4 \Omega^{2}+1}-1\right)^{3} \\
\mathcal{V}_{\Omega}^{T M} & =\frac{L^{2}}{24 \pi a^{2}}\left(3 \xi \Omega^{2}-9 \Omega^{2}+4-\left(2 \Omega^{2}-\xi+1\right) \sqrt{4 \Omega^{2}-2 \xi+2}\right) .
\end{aligned}
$$

Therefore, the number of eigenvalues $\mathcal{N}^{s}(\Omega)$ less than some $\Omega$ is given asymptotically by

$$
\mathcal{N}^{T E}(\Omega), \quad \mathcal{N}^{T M}(\Omega)=\frac{L^{2}}{12 \pi a^{2}} \Omega^{3}-\frac{L^{2}}{8 \pi a^{2}} \Omega^{2}+O(\Omega)
$$

where, remarkably, the asymptotic expressions for the $T E$ and $T M$ modes are identical to order $O(\Omega)$. The result then follows.

Thus in the large $L$ continuum limit the density of states for $T E$ and $T M$ modes behaves like:

$$
\frac{d \mathcal{N}^{s}}{d \Omega} \simeq \frac{L^{2}}{4 \pi a^{2}}\left(\Omega^{2}-\Omega\right)
$$

The expression

$$
\mathcal{E}(\sigma)=\frac{\hbar c_{0}}{2 a \sqrt{\kappa_{0}}} \int_{0}^{\infty} \Omega e^{-\sigma \Omega}\left\{\frac{d \mathcal{N}^{T E}}{d \Omega}+\frac{d \mathcal{N}^{T M}}{d \Omega}\right\} d \Omega \simeq \frac{\hbar c_{0} L^{2}}{\pi \sqrt{\kappa_{0}} a^{3}}\left(\frac{3}{2 \sigma^{4}}\right)
$$

indicates approximately how the large system energy grows with $L$ as $\sigma \rightarrow 0^{+}$. It is of interest to compare this with the $\sigma$ behavior of $\widehat{\mathcal{P}}_{3}^{0,1}\left[F_{\sigma}^{T E}\right]$ and $\mathcal{P}_{3}^{0}\left[F_{\sigma}^{T M}\right]$ derived from (C.7) and (C.12), respectively, in Appendix C. To leading order in $\sigma$ these yield for both values of $s$ :

$$
\widehat{\mathcal{P}}_{3}^{0,1}\left[F_{\sigma}^{T E}\right], \quad \mathcal{P}_{3}^{0}\left[F_{\sigma}^{T M}\right] \simeq \frac{6}{\sigma^{4}}
$$

and a large $L$ energy growth

$$
\mathcal{E}(\sigma)=\frac{\hbar c_{0} L^{2}}{8 \pi a^{3} \sqrt{\kappa_{0}}}\left(\widehat{\mathcal{P}}_{3}^{0,1}\left[F_{\sigma}^{T E}\right]+\mathcal{P}_{3}^{0}\left[F_{\sigma}^{T M}\right]\right) \simeq \frac{\hbar c_{0} L^{2}}{\pi \sqrt{\kappa_{0}} a^{3}}\left(\frac{3}{2 \sigma^{4}}\right)
$$

in precise agreement with (8.1). Thus, the compensating singular parts $\mathcal{P}_{m}^{n}\left[F_{\sigma}^{s}\right], \widehat{\mathcal{P}}_{m}^{n, n_{0}}\left[F_{\sigma}^{s}\right]$ that arise are determined solely by the dependence of the eigenspectrum on the geometry of the physical system. As $\mathcal{V}_{\Omega}^{s} \rightarrow \infty$ and $L \rightarrow \infty$ for fixed $a<L$, the cross-section of the guide grows without bound but the 
inhomogeneous dielectric retains its profile. Thus one may identify in this compensation a one-parameter family of "large volume guides" containing a smooth inhomogeneous medium. When the parameter $a$ also grows to infinity along with $L$ the medium approaches a homogeneous dielectric with relative permittivity $\kappa_{0}$. Since $\sigma$ is dimensionless and the perfectly conducting boundary conditions are maintained in all limiting processes, there is no compelling reason to interpret the regularization process in terms of a physical variation of these conditions with mode frequency.

\section{Conclusions}

In this article, a number of related aspects associated with the problem of determining the ground state expectation values of the electromagnetic quantum field energy and stress in a smooth inhomogeneous polarizable medium have been addressed. After reflecting on the approach taken by Lifshitz et al. and subsequent attempts by some others to calculate such quantities we have pointed out the need to accommodate the relevance of classical mechanical material stresses that must inevitably arise when a physical material continuum is constrained in space in any way. A description of classical mechanical stress induced in incompressible media in static equilibrium with external constraints was presented in terms of the Cauchy Euclidean stress tensor field for a general body. Any attempt to confront contributions to this tensor from theoretical predictions of electromagnetic quantum induced dielectric stresses with experiment should take the material constitutive properties of the medium into account.

Given these general requirements the article has concentrated attention on a specific system composed of a particular meta-material confined in an infinitely long perfectly conducting open waveguide. The dielectric material has been chosen to have an anisotropic and smooth inhomogeneous permittivity that enables one to deduce from the source free Maxwell equations a complete set of electromagnetic eigen-modes and eigen-frequencies analytically. Since these spectral values inhibit propagating harmonic modes in the guide the Lifshitz theory, based on asymptotic scattering states, appears problematic so we have been led to an alternative regularization scheme, based on the Euler-Maclaurin summation formula, for estimating quantum expectation values of electromagnetic energy and stress in the dielectric containing only evanescent eigen-modes.

A detailed exploration of the conditions required for the application of this scheme has been given and shown analytically to yield finite regularized values for the ground state expectation value of the electromagnetic energy in both a constant permittivity and particular inhomogeneous ENZ dielectric. Precise criteria have been given for the general applicability of this scheme used to estimate quantum expectation values together with bounds on the estimate. It has been shown how these criteria can be exploited to construct a general numerical scheme based on earlier work in [29] and benchmarked by comparison with the exact analytic results 
for the Casimir energy per unit area for parallel conducting plates in a vacuum. The excellent agreement between the numerical and analytic estimates augers well for applications of the numerical approach to more general inhomogeneous systems where analytic methods are not available. The article also discusses the EulerMaclaurin regularization of the ground state expectation values of the integrated electromagnetic local stress components in the ENZ medium and how they can be computed numerically.

There remain a number of important effects that have been ignored in this article. These include finite temperature corrections, finite guide conductivity corrections, effects due to spatial and temporal frequency dispersion in the medium, and contributions from material vibration, both classical and quantum. The former as noted will yield stresses from the mechanical constitutive properties of the dielectric and play a role in maintaining static equilibrium configurations in any experimental attempt to detect the quantum induced stresses in the presence of gravitational fields. The considerations in Sec. 2 suggest that experiments with smooth inhomogeneous dielectrics in free-fall may offer a possible environment for detecting quantum induced stresses using the phenomenon of photoelasticity.

By focusing on the spectral properties of a particular ENZ type of medium with a smooth inhomogeneous permittivity in an open guide it has been shown in some detail how finite energy and stress expectation values can be obtained from a welldefined regularization scheme. This has been developed into a numerical procedure that promises wide applicability to systems that are beyond an analytic treatment and offers a new approach to estimate the significance of quantum induced pressures with error estimates based on a generalization of the Euler-Maclaurin summation formula.

\section{Acknowledgments}

The authors are most grateful to Vadim Cheianov for useful conversations. As members of the ALPHA-X collaboration and the Cockcroft Institute of Accelerator Science and Technology, they are also grateful for support from EPSRC (EP/J018171/) and STFC (ST/G008248/1).

\section{Appendix A. General Formulae for Modes in Terms of the Gaussian Hypergeometric Function}

The differential equation

$$
\frac{d^{2}}{d Z^{2}} Y_{\theta, \ell}(Z)+\left(\frac{\Omega_{\theta, \ell}^{2}}{\cosh ^{2} Z} \theta\right) Y_{\theta, \ell}(Z)=0
$$

where

$$
\Omega_{\theta, \ell}^{2} \equiv(\ell+\sqrt{\theta})(\ell+\sqrt{\theta}+1)
$$


has the general (complex) solution

$$
\begin{aligned}
Y_{\theta, \ell}(Z)= & \frac{C_{1}}{\sqrt{\sinh (2 Z)}}(2 \cosh (2 Z)-2)^{3 / 4}(2 \cosh (2 Z)+2)^{1 / 4}(\cosh Z)^{-(\ell+\sqrt{\theta})} \\
& \times \mathcal{G}_{\theta, \ell}^{(-), 1}(Z)+C_{2} \mathcal{G}_{\theta, \ell}^{(+), 3}(Z)(\sinh 2 Z)^{3 / 2}(\cosh Z)^{\ell+\sqrt{\theta}}
\end{aligned}
$$

with arbitrary complex constants $C_{1}, C_{2}$ and where

$$
\mathcal{G}_{\theta, \ell}^{( \pm), \alpha}(Z) \equiv{ }_{2} F_{1}\left(\left[\frac{1 \pm \ell}{2}, \frac{1 \pm \ell \pm 2 \sqrt{\theta}}{2}\right],\left[\frac{\alpha}{2} \pm \sqrt{\theta} \pm \ell\right] ; \frac{1+\cosh (2 Z)}{2}\right)
$$

in terms of the Gaussian hypergeometric function ${ }_{2} F_{1}([a, b],[c] ; \zeta)$. With real $\theta>0$ and all branch cuts drawn on the negative real $Z$ axis, define, for real $Z$ the real valued expression:

$$
\begin{aligned}
\widehat{Y}_{\theta, \ell}(Z)= & \frac{1}{\sqrt{\sinh (2 Z)}}(2 \cosh (2 Z)-2)^{3 / 4}(2 \cosh (2 Z)+2)^{1 / 4} \\
& \times(\cosh Z)^{-(\ell+\sqrt{\theta})} \mathcal{G}_{\theta, \ell}^{(-), 1}(Z) .
\end{aligned}
$$

Then, a complete set $\left\{\mathcal{Z}_{\theta, \ell}(Z)\right\}$ of real continuous normalizable functions, regular for all real $Z$, are defined, for $\ell$ odd $(\ell=1,3,5, \ldots)$, by

$$
\mathcal{Z}_{\theta, \ell}(Z)= \begin{cases}\widehat{Y}_{\theta, \ell}(Z), & Z \geq 0 \\ -\widehat{Y}_{\theta, \ell}(-Z), & Z \leq 0\end{cases}
$$

and for $\ell$ even $(\ell=0,2,4, \ldots)$, by

$$
\mathcal{Z}_{\theta, \ell}(Z)= \begin{cases}i \widehat{Y}_{\theta, \ell}(Z), & Z \geq 0 \\ -\widehat{Y}_{\theta, \ell}(Z), & Z \leq 0\end{cases}
$$

These functions are used to construct the $\left\{\mathcal{Y}_{\mathcal{N}_{s}}^{s}(Z)\right\}$ that enter into the expressions for the pre-potential modes for fields in the dielectric guide with a permittivity profile given by (3.10):

$$
\mathcal{Y}_{\mathcal{N}_{s}}^{s}(Z) \equiv \mathcal{C}_{\mathcal{N}_{s}}^{s} \mathcal{Z}_{\theta, \ell^{s}}(Z)
$$

for normalization constants $\mathcal{C}_{\mathcal{N}_{s}}^{s}$, where $\mathcal{N}_{s} \equiv\left\{n_{x}, n_{y}, \ell\right\}$,

$$
\begin{aligned}
& \theta^{T E}=\pi^{2} a^{2}\left(\frac{n_{x}^{2}}{L_{x}^{2}}+\frac{n_{y}^{2}}{L_{y}^{2}}\right) \\
& \theta^{T M}=\pi^{2} a^{2}\left(\frac{n_{x}^{2}}{L_{x}^{2}}+\frac{n_{y}^{2}}{L_{y}^{2}}\right)+1
\end{aligned}
$$

and $n_{x}, n_{y} \in\{0,1,2, \ldots\}$ (see Sec. 3). The functions $\left\{\mathcal{Y}_{\mathcal{N}_{s}}^{s}(Z)\right\}$ coincide with the functions constructed by a recurrence process in (3.13) up to overall normalization but have the advantage of being explicitly given for all $\mathcal{N}_{s}$ in terms of a finite series involving hyperbolic functions. The normalization constants are given in (4.10). 


\section{Appendix B. Regularization Scheme for a Homogeneous Polarizable Medium}

\section{B.1. Regularized energy}

In this section, we outline the details involved in calculating the quantum expectation value of the electromagnetic ground state energies (6.6) and (6.9) in a homogeneous dielectric contained in a perfectly conducting cuboid cavity (with $\left.L_{x}=L_{y}=L \gg a\right)$ in terms of integrals in $\Gamma_{M}^{n}\left[F_{\sigma}^{s}\right]$. The dimensionless auxiliary function $F_{\sigma}^{s}$ is

$$
F_{\sigma}^{s}\left(n_{z}\right)=\int_{n_{z}^{2}}^{\infty} \sqrt{u} \exp (-\sigma \pi \sqrt{u}) d u .
$$

For this case $M=2$ in the Euler-Maclaurin expansion and $\lim _{\sigma \rightarrow 0^{+}} \varepsilon_{2}^{n}\left[F_{\sigma}^{s}\right]=$ 0 for all $n \geq 0$. The results for the regularized sums are then exactly $\beta_{2}^{n, s}$ for $s \in\{T E, T M\}$ and for all $n \geq 0$. The dimensionless auxiliary function $F_{\sigma}^{s}$ can be evaluated analytically:

$$
\begin{aligned}
F_{\sigma}^{s}\left(n_{z}\right) & =2 \int_{n_{z}}^{\infty} y^{2} e^{-\sigma \pi y} d y=\frac{2}{\pi^{2}} \frac{d^{2}}{d \sigma^{2}}\left(\int_{n_{z}}^{\infty} e^{-\sigma \pi y} d y\right)=\frac{2}{\pi^{2}} \frac{d^{2}}{d \sigma^{2}}\left(\frac{e^{-\sigma \pi n_{z}}}{\pi \sigma}\right) \\
& =\left(\frac{4}{\pi^{2} \sigma^{2}}+\frac{4 n_{z}}{\pi \sigma}+2 n_{z}^{2}\right) \frac{e^{-\sigma \pi n_{z}}}{\pi \sigma}
\end{aligned}
$$

yielding

$$
\begin{aligned}
\int_{n}^{\infty} F_{\sigma}^{s}(x) d x & =\int_{n}^{\infty}\left(\frac{4}{\pi^{2} \sigma^{2}}+\frac{4 x}{\pi \sigma}+2 x^{2}\right) \frac{e^{-\sigma \pi x}}{\pi \sigma} d x \\
& =\left(\frac{12}{\pi^{2} \sigma^{2}}+\frac{8 n}{\pi \sigma}+2 n^{2}\right) \frac{e^{-\sigma \pi n}}{\pi^{2} \sigma^{2}}
\end{aligned}
$$

Thus

$$
\begin{array}{rlrl}
F_{\sigma}^{T M}(0) & =\frac{4}{\pi^{3} \sigma^{3}} & F_{\sigma}^{T E}(1) & =\left(\frac{4}{\pi^{2} \sigma^{2}}+\frac{4}{\pi \sigma}+2\right) \frac{e^{-\sigma \pi}}{\pi \sigma} \\
\int_{0}^{\infty} F_{\sigma}^{T M}(x) d x & =\frac{12}{\pi^{4} \sigma^{4}} \quad \int_{1}^{\infty} F_{\sigma}^{T E}(x) d x=\left(\frac{12}{\pi^{2} \sigma^{2}}+\frac{8}{\pi \sigma}+2\right) \frac{e^{-\sigma \pi}}{\pi^{2} \sigma^{2}} .
\end{array}
$$

Using (5.2):

$$
S_{2}(k)\left[F_{\sigma}^{s}\right]=\left(\frac{1}{180}-\frac{\sigma \pi k}{90}-\frac{k^{2}}{6}+\frac{\sigma^{2} \pi^{2} k^{2}}{360}\right) e^{-\sigma \pi k}
$$

yielding

$$
S_{2}(0)\left[F_{\sigma}^{T M}\right]=\frac{1}{180} \quad \text { and } \quad S_{2}(1)\left[F_{\sigma}^{T E}\right]=\left(-\frac{29}{180}-\frac{\sigma \pi}{90}+\frac{\sigma^{2} \pi^{2}}{360}\right) e^{-\sigma \pi} .
$$

Then (5.7) gives

$$
\Gamma_{2}^{1}\left[F_{\sigma}^{T E}\right]=\left(\frac{12}{\pi^{4} \sigma^{4}}+\frac{10}{\pi^{3} \sigma^{3}}+\frac{4}{\pi^{2} \sigma^{2}}+\frac{1}{\pi \sigma}+\frac{29}{180}-\frac{\pi \sigma}{90}+\frac{\pi^{2} \sigma^{2}}{360}\right) \frac{e^{-\sigma \pi}}{\pi^{2} \sigma^{2}}
$$


or taking the series expansion with respect to $\sigma$ :

$$
\Gamma_{2}^{1}\left[F_{\sigma}^{T E}\right]=\frac{12}{\pi^{4} \sigma^{4}}-\frac{2}{\pi^{3} \sigma^{3}}-\frac{1}{180}+\frac{\pi^{3} \sigma^{3}}{756}+O\left(\sigma^{4}\right),
$$

and

$$
\Gamma_{2}^{0}\left[F_{\sigma}^{T M}\right]=\frac{12}{\pi^{4} \sigma^{4}}+\frac{2}{\pi^{3} \sigma^{3}}-\frac{1}{180} .
$$

Hence

$$
\begin{aligned}
& \mathcal{P}_{2}^{1}\left[F_{\sigma}^{T E}\right]=\frac{12}{\pi^{4} \sigma^{4}}-\frac{2}{\pi^{3} \sigma^{3}}, \quad \beta_{2}^{1, T E}=-\frac{1}{180} \\
& \mathcal{P}_{2}^{0}\left[F_{\sigma}^{T M}\right]=\frac{12}{\pi^{4} \sigma^{4}}+\frac{2}{\pi^{3} \sigma^{3}}, \quad \beta_{2}^{0, T M}=-\frac{1}{180}
\end{aligned}
$$

and (5.13) yields the regularized sums

$$
\begin{aligned}
& \lim _{\sigma \rightarrow 0^{+}}\left(\sum_{n_{z}=1}^{\infty} F_{\sigma}^{T E}\left(n_{z}\right)-\frac{12}{\pi^{4} \sigma^{4}}+\frac{2}{\pi^{3} \sigma^{3}}\right)=-\frac{1}{180} \\
& \lim _{\sigma \rightarrow 0^{+}}\left(\sum_{n_{z}=0}^{\infty} F_{\sigma}^{T M}\left(n_{z}\right)-\frac{12}{\pi^{4} \sigma^{4}}-\frac{2}{\pi^{3} \sigma^{3}}\right)=-\frac{1}{180} .
\end{aligned}
$$

\section{B.2. Regularized integrated stress}

The computation of quantum induced electromagnetic integrated stress at any section (parallel to the faces) of the cuboid involves the analysis of $\Gamma_{M}^{n}\left[\bar{F}_{\sigma}^{s}\right]$, in terms of the dimensionless auxiliary function

$$
\bar{F}_{\sigma}^{s}\left(n_{z}\right)=\int_{n_{z}^{2}}^{\infty} \frac{n_{z}^{2}}{\sqrt{u}} \exp (-\sigma \pi \sqrt{u}) d u .
$$

In this case, $M=2$ in the Euler-Maclaurin expansion and $\lim _{\sigma \rightarrow 0^{+}} \varepsilon_{2}^{n}\left[\bar{F}_{\sigma}^{s}\right]=0$ for all $n \geq 0$. The results for the regularized sums are then exactly $\beta_{2}^{n, s}$ for $s \in$ $\{T E, T M\}$ and for all $n \geq 0$. The dimensionless auxiliary function $\bar{F}_{\sigma}^{s}\left(n_{z}\right)$ can be evaluated analytically:

$$
\bar{F}_{\sigma}^{s}\left(n_{z}\right)=2 n_{z}^{2} \int_{n_{z}}^{\infty} e^{-\sigma \pi y} d y=\frac{2 n_{z}^{2} e^{-\sigma \pi n_{z}}}{\pi \sigma}
$$

yielding

$$
\int_{n}^{\infty} \bar{F}_{\sigma}^{s}(x) d x=\frac{2}{\pi \sigma} \int_{n}^{\infty} x^{2} e^{-\sigma \pi x} d x=\left(\frac{4}{\pi^{2} \sigma^{2}}+\frac{4 n}{\pi \sigma}+2 n^{2}\right) \frac{e^{-\sigma \pi n}}{\pi^{2} \sigma^{2}} .
$$

Thus

$$
\begin{array}{rlrl}
\bar{F}_{\sigma}^{T M}(0) & =0 & \bar{F}_{\sigma}^{T E}(1) & =\frac{2 e^{-\sigma \pi}}{\pi \sigma} \\
\int_{0}^{\infty} \bar{F}_{\sigma}^{T M}(x) d x & =\frac{4}{\pi^{4} \sigma^{4}} \quad \int_{1}^{\infty} \bar{F}_{\sigma}^{T E}(x) d x & =\left(\frac{4}{\pi^{2} \sigma^{2}}+\frac{4}{\pi \sigma}+2\right) \frac{e^{-\sigma \pi}}{\pi^{2} \sigma^{2}} .
\end{array}
$$


Using (5.2)

$$
S_{2}(k)\left[\bar{F}_{\sigma}^{s}\right]=\left(\frac{k}{3 \sigma \pi}+\frac{1}{60}-\frac{\sigma \pi k}{60}-\frac{k^{2}}{6}+\frac{\sigma^{2} \pi^{2} k^{2}}{360}\right) e^{-\sigma \pi k}
$$

gives

$$
S_{2}(0)\left[\bar{F}_{\sigma}^{T M}\right]=\frac{1}{60} \quad \text { and } \quad S_{2}(1)\left[\bar{F}_{\sigma}^{T E}\right]=\left(\frac{1}{3 \sigma \pi}-\frac{3}{20}-\frac{\sigma \pi}{60}+\frac{\sigma^{2} \pi^{2}}{360}\right) e^{-\sigma \pi} .
$$

Hence from (5.7)

$$
\Gamma_{2}^{1}\left[\bar{F}_{\sigma}^{T E}\right]=\left(\frac{4}{\pi^{4} \sigma^{4}}+\frac{4}{\pi^{3} \sigma^{3}}+\frac{2}{\pi^{2} \sigma^{2}}-\frac{2}{3 \sigma \pi}+\frac{3}{20}+\frac{\sigma \pi}{60}-\frac{\sigma^{2} \pi^{2}}{360}\right) e^{-\sigma \pi}
$$

or taking the series expansion with respect to $\sigma$ :

$$
\Gamma_{2}^{1}\left[\bar{F}_{\sigma}^{T E}\right]=\frac{4}{\pi^{4} \sigma^{4}}-\frac{1}{60}+\frac{\pi^{3} \sigma^{3}}{504}+O\left(\sigma^{4}\right)
$$

and

Therefore

$$
\Gamma_{2}^{0}\left[\bar{F}_{\sigma}^{T M}\right]=\frac{4}{\pi^{4} \sigma^{4}}-\frac{1}{60}
$$

$$
\begin{array}{rlrl}
\mathcal{P}_{2}^{1}\left[\bar{F}_{\sigma}^{T E}\right] & =\frac{4}{\pi^{4} \sigma^{4}}, & \beta_{2}^{1, T E} & =-\frac{1}{60} \\
\mathcal{P}_{2}^{0}\left[\bar{F}_{\sigma}^{T M}\right]=\frac{4}{\pi^{4} \sigma^{4}}+\frac{2}{\pi^{3} \sigma^{3}}, & \beta_{2}^{0, T M} & =-\frac{1}{60}
\end{array}
$$

and (5.13) yields the regularized sums

$$
\begin{aligned}
& \lim _{\sigma \rightarrow 0^{+}}\left(\sum_{n_{z}=1}^{\infty} \bar{F}_{\sigma}^{T E}\left(n_{z}\right)-\frac{4}{\pi^{4} \sigma^{4}}\right)=-\frac{1}{60} \\
& \lim _{\sigma \rightarrow 0^{+}}\left(\sum_{n_{z}=0}^{\infty} \bar{F}_{\sigma}^{T M}\left(n_{z}\right)-\frac{4}{\pi^{4} \sigma^{4}}\right)=-\frac{1}{60} .
\end{aligned}
$$

\section{Appendix C. Regularization Scheme for a Smooth Inhomogeneous Polarizable Medium}

In this section, we outline the details involved in calculating the TE and TM parts of the quantum expectation value of the electromagnetic ground state energy (7.1) of the open guide (with $L_{x}=L_{y}=L \gg a$ ) containing the medium with the permittivity profile given by (3.10), in terms of certain $\sigma$-dependent integrals labeled as follows:

$$
\mathcal{I}_{(a, b)}^{p, q} \equiv \int_{a}^{b} \frac{u^{p / 2}}{(1+4 u)^{q / 2}} \exp (-\sigma \sqrt{u}) d u .
$$

From (5.7), the behavior of $\Gamma_{m}^{n}\left[F_{\sigma}^{s}\right]$ as a function of $m$ necessitates a value of $m \geq 1$ for (5.10) to be satisfied. However, based on the behavior of $T_{m}^{n}$ as a function of $m$, in the following we choose $m=3$ so that the values of $\beta_{m}^{n, s}$ determined by $\Gamma_{m}^{n}\left[F_{\sigma}^{s}\right]$ can be calculated to 6 significant figures. 


\section{C.1. Contribution from TE modes}

For the TE mode spectrum the dimensionless auxiliary function is:

$$
F_{\sigma}^{T E}(\ell)=\int_{\ell(\ell+1)}^{\infty} \sqrt{u}\left(1-\frac{2 \ell+1}{\sqrt{1+4 u}}\right) \exp (-\sigma \sqrt{u}) d u .
$$

With $n=0$, the Euler-Maclaurin regularization procedure outlined in Sec. 5 does not yield a finite result, since terms in $S_{m}(n)\left[F_{\sigma}^{T E}\right]$ diverge as $n \rightarrow 0$. However a regularization can proceed for $n=1$ with $\Gamma_{3}^{1}\left[F_{\sigma}^{T E}\right]$ defined by (5.7) and from (5.17):

$$
\widehat{\Gamma}_{3}^{0,1}\left[F_{\sigma}^{T E}\right]=F_{\sigma}^{T E}(0)+\Gamma_{3}^{1}\left[F_{\sigma}^{T E}\right] .
$$

First note that

$$
F_{\sigma}^{T E}(1)=\mathcal{I}_{(2, \infty)}^{1,0}-3 \mathcal{I}_{(2, \infty)}^{1,1}=\mathcal{I}_{(0, \infty)}^{1,0}-\mathcal{I}_{(0,2)}^{1,0}-3 \mathcal{I}_{(0, \infty)}^{1,1}+3 \mathcal{I}_{(0,2)}^{1,1} .
$$

To compute the integral term in (5.7), we shall use the identity

$$
\int_{1}^{\infty} F_{\sigma}^{T E}(x) d x=\int_{0}^{\infty} F_{\sigma}^{T E}(x) d x-\int_{0}^{1} F_{\sigma}^{T E}(x) d x .
$$

For any integrable function $f(x, u)$, one has, by reversing the order of integration,

$$
\int_{0}^{\infty}\left(\int_{x(x+1)}^{\infty} f(x, u) d u\right) d x=\int_{0}^{\infty}\left(\int_{0}^{u_{0}} f(x, u) d x\right) d u
$$

where $u_{0}=-\frac{1}{2}+\frac{1}{2} \sqrt{4 u+1}$. Thus

$$
\begin{aligned}
\int_{0}^{\infty} F_{\sigma}^{T E}(x) d x & =\int_{0}^{\infty}\left[\frac{\sqrt{u}}{2}(\sqrt{1+4 u}-1)-\frac{u^{3 / 2}}{\sqrt{1+4 u}}\right] \exp (-\sigma \sqrt{u}) d u \\
& =\frac{1}{2} \mathcal{I}_{(0, \infty)}^{1,-1}-\frac{1}{2} \mathcal{I}_{(0, \infty)}^{1,0}-\mathcal{I}_{(0, \infty)}^{3,1}
\end{aligned}
$$

since the integration over $x$ can be performed analytically. Similarly, from

$$
\begin{aligned}
\int_{0}^{1}\left(\int_{x(x+1)}^{\infty} f(x, u) d u\right) d x & =\int_{0}^{1}\left(\int_{0}^{\infty} f(x, u) d u-\int_{0}^{x(x+1)} f(x, u) d u\right) d x \\
& =\int_{0}^{\infty}\left(\int_{0}^{1} f(x, u) d x\right) d u-\int_{0}^{2}\left(\int_{u_{0}}^{1} f(x, u) d x\right) d u
\end{aligned}
$$

one has

$$
\int_{0}^{1} F_{\sigma}^{T E}(x) d x=\mathcal{I}_{(0, \infty)}^{1,0}-\frac{3}{2} \mathcal{I}_{(0,2)}^{1,0}+\frac{1}{2} \mathcal{I}_{(0,2)}^{1,-1}-2 \mathcal{I}_{(0, \infty)}^{1,1}+2 \mathcal{I}_{(0,2)}^{1,1}-\mathcal{I}_{(0,2)}^{3,1},
$$

since again, the integration over $x$ can be performed analytically. Thus

$$
\begin{aligned}
\int_{1}^{\infty} F_{\sigma}^{T E}(x) d x= & \frac{1}{2} \mathcal{I}_{(0, \infty)}^{1,-1}-\frac{3}{2} \mathcal{I}_{(0, \infty)}^{1,0}+2 \mathcal{I}_{(0, \infty)}^{1,1}-\mathcal{I}_{(0, \infty)}^{3,1}+\frac{3}{2} \mathcal{I}_{(0,2)}^{1,0} \\
& -\frac{1}{2} \mathcal{I}_{(0,2)}^{1,-1}-2 \mathcal{I}_{(0,2)}^{1,1}+\mathcal{I}_{(0,2)}^{3,1}
\end{aligned}
$$


using (C.2). Finally, from with $m=3$

$$
\begin{aligned}
S_{3}(1)\left[F_{\sigma}^{T E}\right]= & -\frac{1}{6} \mathcal{I}_{(0, \infty)}^{(1,1)}+\frac{1}{6} \mathcal{I}_{(0,2)}^{(1,1)}-\frac{223 \sqrt{2}}{107520} \exp (-\sigma \sqrt{2})+\frac{15}{3584} \sigma \exp (-\sigma \sqrt{2}) \\
& +\frac{\sqrt{2}}{6720} \sigma^{2} \exp (-\sigma \sqrt{2})-\frac{1}{8960} \sigma^{3} \exp (-\sigma \sqrt{2}) .
\end{aligned}
$$

Therefore, using (5.7), one obtains

$$
\begin{aligned}
\Gamma_{3}^{1}\left[F_{\sigma}^{T E}\right]= & \mathcal{I}_{(0,2)}^{1,0}-\mathcal{I}_{(0, \infty)}^{1,0}+\frac{2}{3} \mathcal{I}_{(0, \infty)}^{1,1}-\frac{2}{3} \mathcal{I}_{(0,2)}^{1,1}+\frac{1}{2} \mathcal{I}_{(0, \infty)}^{1,-1}-\frac{1}{2} \mathcal{I}_{(0,2)}^{1,-1}-\mathcal{I}_{(0, \infty)}^{3,1} \\
& +\mathcal{I}_{(0,2)}^{3,1}+\frac{223 \sqrt{2}}{107520} \exp (-\sigma \sqrt{2})-\frac{15}{3584} \sigma \exp (-\sigma \sqrt{2}) \\
& -\frac{\sqrt{2}}{6720} \sigma^{2} \exp (-\sigma \sqrt{2})+\frac{1}{8960} \sigma^{3} \exp (-\sigma \sqrt{2})
\end{aligned}
$$

Since

$$
F_{\sigma}^{T E}(0)=\mathcal{I}_{(0, \infty)}^{1,0}-\mathcal{I}_{(0, \infty)}^{1,1}
$$

it follows from (5.17) that:

$$
\begin{aligned}
\widehat{\Gamma}_{3}^{0,1}\left[F_{\sigma}^{T E}\right]= & \mathcal{I}_{(0,2)}^{1,0}-\frac{1}{3} \mathcal{I}_{(0, \infty)}^{1,1}-\frac{2}{3} \mathcal{I}_{(0,2)}^{1,1}+\frac{1}{2} \mathcal{I}_{(0, \infty)}^{1,-1}-\frac{1}{2} \mathcal{I}_{(0,2)}^{1,-1}-\mathcal{I}_{(0, \infty)}^{3,1} \\
& +\mathcal{I}_{(0,2)}^{3,1}+\frac{223 \sqrt{2}}{107520} \exp (-\sigma \sqrt{2})-\frac{15}{3584} \sigma \exp (-\sigma \sqrt{2}) \\
& -\frac{\sqrt{2}}{6720} \sigma^{2} \exp (-\sigma \sqrt{2})+\frac{1}{8960} \sigma^{3} \exp (-\sigma \sqrt{2}) .
\end{aligned}
$$

The infinite range integrals in (C.4) can be evaluated analytically with the results:

$$
\begin{aligned}
& \mathcal{I}_{(0, \infty)}^{1,1}=\frac{\pi}{4} \frac{d \mathcal{K}}{d \sigma}, \quad \mathcal{I}_{(0, \infty)}^{1,-1}=-\pi \frac{d^{2}}{d \sigma^{2}}\left(\frac{\mathcal{K}}{\sigma}\right) \\
& \mathcal{I}_{(0, \infty)}^{3,1}=\frac{d^{2}}{d \sigma^{2}} \mathcal{I}_{(0, \infty)}^{1,1}=\frac{\pi}{4} \frac{d^{3} \mathcal{K}}{d \sigma^{3}}
\end{aligned}
$$

where

$$
\mathcal{K}(\sigma) \equiv Y_{1}\left(\frac{\sigma}{2}\right)-H_{1}\left(\frac{\sigma}{2}\right)
$$

in terms of the first order Bessel function of the second kind $Y_{1}(x)$ and the first order Struve function $H_{1}(x)$. Series expansions of the finite range integrals in (C.4) around $\sigma=0$ can be evaluated without computing their exact analytic form. For example, ${ }^{\mathrm{e}}$

$$
\mathcal{I}_{(0,2)}^{1,0}=\int_{0}^{2} \sqrt{u} e^{-\sigma \sqrt{u}} d u=\int_{0}^{2}(\sqrt{u}-\sigma u) d u+O\left(\sigma^{2}\right)
$$

e In this case the integral can be computed analytically and the series expansion of the result about $\sigma=0$ is in agreement with the integration of the series expansion of the integrand about $\sigma=0$. 
S. Goto, R. W. Tucker \& T. J. Walton

$$
\begin{aligned}
& =\left[\frac{2}{3} u^{3 / 2}-\frac{1}{2} \sigma u^{2}\right]_{u=0}^{2}+O\left(\sigma^{2}\right) \\
& =\frac{4 \sqrt{2}}{3}-2 \sigma+O\left(\sigma^{2}\right)
\end{aligned}
$$

since $\sqrt{u} e^{-\sigma \sqrt{u}}=\sqrt{u}-\sigma u+O\left(\sigma^{2}\right)$. Thus, using (C.5), the series expansion of (C.4) around $\sigma=0$, is calculated as:

$$
\begin{aligned}
\widehat{\Gamma}_{3}^{0,1}\left[F_{\sigma}^{T E}\right]= & \frac{6}{\sigma^{4}}+\frac{1}{24 \sigma^{2}}-\frac{1}{384} \ln (\sigma)-\frac{5}{1536}-\frac{5339 \sqrt{2}}{35840} \\
& -\frac{\gamma}{384}-\frac{43}{768} \ln (2)+\frac{47}{384} \ln (4+3 \sqrt{2})+O\left(\sigma^{2}\right)
\end{aligned}
$$

in terms of Euler's constant $\gamma$. Using (5.18), this gives

$$
\begin{aligned}
\widehat{\mathcal{P}}_{3}^{0,1}\left[F_{\sigma}^{T E}\right] & =\frac{6}{\sigma^{4}}+\frac{1}{24 \sigma^{2}}-\frac{1}{384} \ln (\sigma) \\
\widehat{\beta}_{3}^{0,1, T E} & =-\frac{5}{1536}-\frac{5339 \sqrt{2}}{35840}-\frac{\gamma}{384}-\frac{43}{768} \ln (2)+\frac{47}{384} \ln (4+3 \sqrt{2}) \\
& =0.00393263
\end{aligned}
$$

to 6 significant figures. The term $T_{m}(1, \infty)\left[F_{\sigma}^{T E}\right]$ cannot be evaluated analytically but, using (5.14), it can be bounded. For $m=3$, a bound is $\lim _{\sigma \rightarrow 0^{+}} \varepsilon_{3}^{1}\left[F_{\sigma}^{T E}\right] \leq$ $6.40 \times 10^{-6}$, yielding the relative error

$$
\frac{\lim _{\sigma \rightarrow 0^{+}} \varepsilon_{3}^{1}\left[F_{\sigma}^{T E}\right]}{\left|\widehat{\beta}_{3}^{0,1, T E}\right|} \leq 1.623 \times 10^{-3}
$$

Thus from (5.16):

$$
\begin{aligned}
\lim _{\sigma \rightarrow 0^{+}} & \left(\sum_{\ell=0}^{\infty} F_{\sigma}^{T E}(\ell)-\frac{6}{\sigma^{4}}-\frac{1}{24 \sigma^{2}}+\frac{1}{384} \ln (\sigma)\right) \\
= & -\frac{5}{1536}-\frac{5339 \sqrt{2}}{35840}-\frac{\gamma}{384}-\frac{43}{768} \ln (2)+\frac{47}{384} \ln (4+3 \sqrt{2}) \\
& \pm \lim _{\sigma \rightarrow 0^{+}} \varepsilon_{3}^{1}\left[F_{\sigma}^{T E}\right]=0.00393263 \pm 6.40 \times 10^{-6}
\end{aligned}
$$

to 6 significant figures.

\section{C.2. Contribution from TM modes}

For the $T M$ modes the dimensionless auxiliary function is

$$
F_{\sigma}^{T M}(\ell)=\int_{(\ell+1)(\ell+2)}^{\infty} \sqrt{u}\left(1-\frac{2 \ell+1}{\sqrt{1+4 u}}\right) \exp (-\sigma \sqrt{u}) d u .
$$

To analyze $\Gamma_{3}^{0}\left[F_{\sigma}^{T M}\right]$ as defined by (5.7), first note that

$$
F_{\sigma}^{T M}(0)=\mathcal{I}_{(2, \infty)}^{1,0}-\mathcal{I}_{(2, \infty)}^{1,1}=\mathcal{I}_{(0, \infty)}^{1,0}-\mathcal{I}_{(0,2)}^{1,0}-\mathcal{I}_{(0, \infty)}^{1,1}+\mathcal{I}_{(0,2)}^{1,1} .
$$


Next, for any integrable function $f(x, u)$, one has, by reversing the order of integration,

$$
\int_{0}^{\infty}\left(\int_{(x+2)(x+1)}^{\infty} f(x, u) d u\right) d x=\int_{2}^{\infty}\left(\int_{0}^{u_{0}} f(x, u) d x\right) d u
$$

where $u_{0}=-\frac{3}{2}+\frac{1}{2} \sqrt{4 u+1}$. Thus

$$
\begin{aligned}
\int_{0}^{\infty} F_{\sigma}^{T M}(x) d x= & \int_{2}^{\infty}\left[\frac{\sqrt{u}}{2}(\sqrt{1+4 u}-3)-\frac{\sqrt{u}(1+u)}{\sqrt{1+4 u}}+\sqrt{u}\right] \exp (-\sigma \sqrt{u}) d u \\
= & -\frac{1}{2} \mathcal{I}_{(2, \infty)}^{1,0}+\frac{1}{2} \mathcal{I}_{(2, \infty)}^{1,-1}-\mathcal{I}_{(2, \infty)}^{1,1}-\mathcal{I}_{(2, \infty)}^{3,1} \\
= & -\frac{1}{2} \mathcal{I}_{(0, \infty)}^{1,0}+\frac{1}{2} \mathcal{I}_{(0,2)}^{1,0}+\frac{1}{2} \mathcal{I}_{(0, \infty)}^{1,-1}-\frac{1}{2} \mathcal{I}_{(0,2)}^{1,-1}-\mathcal{I}_{(0, \infty)}^{1,1}+\mathcal{I}_{(0,2)}^{1,1} \\
& -\mathcal{I}_{(0, \infty)}^{3,1}+\mathcal{I}_{(0,2)}^{3,1}
\end{aligned}
$$

since the integration over $x$ can be performed analytically. Also, using (5.2),

$$
\begin{aligned}
S_{3}(0)\left[F_{\sigma}^{T M}\right]= & -\frac{1}{6} \mathcal{I}_{(0, \infty)}^{(1,1)}+\frac{1}{6} \mathcal{I}_{(0,2)}^{(1,1)}-\frac{31129 \sqrt{2}}{184320} \exp (-\sigma \sqrt{2}) \\
& -\frac{17}{10240} \sigma \exp (-\sigma \sqrt{2})+\frac{2129 \sqrt{2}}{645120} \sigma^{2} \exp (-\sigma \sqrt{2}) \\
& +\frac{3}{17920} \sigma^{3} \exp (-\sigma \sqrt{2})-\frac{3 \sqrt{2}}{35840} \sigma^{4} \exp (-\sigma \sqrt{2}) .
\end{aligned}
$$

Therefore, from (5.7),

$$
\begin{aligned}
\Gamma_{3}^{0}\left[F_{\sigma}^{T M}\right]= & -\frac{4}{3} \mathcal{I}_{(0, \infty)}^{1,1}+\frac{4}{3} \mathcal{I}_{(0,2)}^{1,1}+\frac{1}{2} \mathcal{I}_{(0, \infty)}^{1,-1}-\frac{1}{2} \mathcal{I}_{(0,2)}^{1,-1}-\mathcal{I}_{(0, \infty)}^{3,1}+\mathcal{I}_{(0,2)}^{3,1} \\
& +\frac{31129 \sqrt{2}}{184320} \exp (-\sigma \sqrt{2})+\frac{17}{10240} \sigma \exp (-\sigma \sqrt{2}) \\
& -\frac{2129 \sqrt{2}}{645120} \sigma^{2} \exp (-\sigma \sqrt{2})-\frac{3}{17920} \sigma^{3} \exp (-\sigma \sqrt{2}) \\
& +\frac{3 \sqrt{2}}{35840} \sigma^{4} \exp (-\sigma \sqrt{2}) .
\end{aligned}
$$

Using (C.5), the series expansion of (C.10) around $\sigma=0$, yields

$$
\begin{aligned}
\Gamma_{3}^{0}\left[F_{\sigma}^{T M}\right]= & \frac{6}{\sigma^{4}}-\frac{23}{24 \sigma^{2}}-\frac{49}{384} \ln (\sigma)-\frac{101}{1536}+\frac{34009 \sqrt{2}}{184320}-\frac{49 \gamma}{384} \\
& +\frac{245}{768} \ln (2)-\frac{49}{384} \ln (4+3 \sqrt{2})+\frac{4}{45} \sigma+O\left(\sigma^{2}\right)
\end{aligned}
$$


From (5.8), this gives

$$
\begin{aligned}
\mathcal{P}_{3}^{0}\left[F_{\sigma}^{T M}\right] & =\frac{6}{\sigma^{4}}-\frac{23}{24 \sigma^{2}}-\frac{49}{384} \ln (\sigma) \\
\beta_{3}^{0, T M} & =-\frac{101}{1536}+\frac{34009 \sqrt{2}}{184320}-\frac{49 \gamma}{384}+\frac{245}{768} \ln (2)-\frac{49}{384} \ln (4+3 \sqrt{2}) \\
& =0.0734902
\end{aligned}
$$

to 6 significant figures. As with the $T E$ modes, the term $T_{m}(0, \infty)\left[F_{\sigma}^{T M}\right]$ cannot be found analytically but, using (5.14), it can be bounded. For $m=3$, a bound is $\lim _{\sigma \rightarrow 0^{+}} \varepsilon_{3}^{0}\left[F_{\sigma}^{T M}\right] \leq 3.17 \times 10^{-5}$, yielding the relative error

$$
\frac{\lim _{\sigma \rightarrow 0^{+}} \varepsilon_{3}^{0}\left[F_{\sigma}^{T M}\right]}{\left|\beta_{3}^{0, T M}\right|} \leq 4.31 \times 10^{-4}
$$

Thus

$$
\begin{aligned}
\lim _{\sigma \rightarrow 0^{+}}\left(\sum_{\ell=0}^{\infty} F_{\sigma}^{T M}(\ell)-\frac{6}{\sigma^{4}}+\frac{23}{24 \sigma^{2}}+\frac{49}{384} \ln (\sigma)\right) \\
=-\frac{101}{1536}+\frac{34009 \sqrt{2}}{184320}-\frac{49 \gamma}{384}+\frac{245}{768} \ln (2)-\frac{49}{384} \ln (4+3 \sqrt{2}) \\
\quad \pm \lim _{\sigma \rightarrow 0^{+}} \varepsilon_{3}^{1}\left[F_{\sigma}^{T M}\right]=0.0734902 \pm 3.17 \times 10^{-5}
\end{aligned}
$$

to 6 significant figures. It should be noted that the relations (C.8) and (C.13) are invariant under $\sigma \mapsto q(\sigma)$.

\section{Appendix D. The Generalized Abel-Plana Formula}

For electromagnetic systems with real eigen-frequencies given by the roots of equations that are not algebraic, an alternative numerical regularization scheme can be constructed based on the Abel-Plana formula instead of (5.1). Suppose such a system has the real positive eigenvalue spectrum $\mathfrak{S}=\left\{\mu_{1}, \ldots, \mu_{r}, \ldots\right\}$ where $\mathfrak{S}$ contains all the ordered roots of $F(\mu)=0$ such that

$$
\Delta(z)=\frac{F^{\prime}(z)}{F(z)}
$$

has simple poles at the elements of $\mathfrak{S}$ with unit residue in the complex $z$-plane. Furthermore, let $f(z)$ be analytic in $z$ for $\operatorname{Re}(z)>\xi, \xi<\mu_{1}$. Then the generalized Abel-Plana formula follows from the Cauchy integral formula:

$$
\sum_{r=1}^{n} f\left(\mu_{r}\right)-\int_{\mu_{1}}^{\mu_{n}} f(x) d x=\frac{1}{2} f\left(\mu_{1}\right)+\frac{1}{2} f\left(\mu_{n}\right)+Q_{y_{0}}(n)-Q_{y_{0}}(1)+\frac{1}{2 i}\left(L_{+}+L_{-}\right)
$$

where

$$
\begin{aligned}
L_{ \pm} & =\mp \int_{\mu_{1}}^{\mu_{n}} f\left(x \pm i y_{0}\right) \psi_{ \pm}\left(x \pm i y_{0}\right) d x \\
Q_{y_{0}}(r) & =\frac{1}{2} \int_{0}^{y_{0}}\left\{f\left(\mu_{r}+i y\right) \psi_{+}\left(\mu_{r}+i y\right)+f\left(\mu_{r}-i y\right) \psi_{-}\left(\mu_{r}-i y\right)\right\} d y
\end{aligned}
$$


with

$$
\psi_{ \pm}(z)=\frac{\Delta(z)}{\pi} \pm i
$$

and any real $y_{0}>0$. This reduces to the Abel-Plana formula (p. 340, 20]) when $F(\mu)=\sin (\pi \mu)$.

Recourse to this generalized Abel-Plana formula offers an alternative to the generalized Euler-Maclaurin formulae based on (5.1) since it is rare that one can find exact solutions for the spectra of electromagnetic modes in a general confined system as the boundary conditions often lead to eigenvalues that are the roots of transcendental equations. Furthermore, the criteria for its implementation as a viable regularization scheme are somewhat more restrictive when dealing with inhomogeneous media since one needs to assess the significance of contributions from integrals over contours in the complex plane.

\section{References}

[1] H. B. G. Casimir, On the attraction between two perfectly conducting plates, Proc. K. Ned. Akad. Wet. 51 (1948) 793-795.

[2] E. M. Lifshitz, The theory of molecular attractive forces between solids, Soviet Phys. JETP 2 (1956) 73-83.

[3] T. G. Philbin, C. Xiong and U. Leonhardt, Casimir stress in an inhomogeneous medium, Ann. Phys. 325 (2010) 579-595.

[4] M. Bordag, G. L. Klimchitskaya, U. Mohideen and V. M. Mostepanenko, Advances in the Casimir Effect (Oxford University Press, Oxford, 2009).

[5] S. Goto, R. W. Tucker and T. J. Walton, Quantum electromagnetic vacuum fluctuations in inhomogeneous dielectric media, Proc. SPIE 8072 (2011) 80720, 7 pp.

[6] U. Leonhardt and W. M. R. Simpson, Exact solution for the Casimir stress in a spherically symmetric medium, Phys. Rev. D 84 (2011) 081701, 5 pp.

[7] S. Goto, R. W. Tucker and T. J. Walton, On the computation of Casimir stresses in open media and Lifshitz theory, J. Phys. A Math. Theor. 46 (2013) 405301.

[8] I. Brevik, Electrostrictive contribution to the Casimir effect in a dielectric sphere, Ann. Phys. 138 (1982) 36-52.

[9] W. M. R. Simpson and S. A. R. Horsley and U. Leonhardt, Divergence of Casimir stress in inhomogeneous media, Phys. Rev. A 87 (2013) 043806.

[10] S. Goto, R. W. Tucker and T. J. Walton, The electrodynamics of inhomogeneous rotating media and the Abraham and Minkowski tensors: Supplementary material, Proc. R. Soc. Lond. Ser. A Math. Phys. Eng. Sci. 467 (2011) 79-98.

[11] A. Alù, M. G. Silveirinha, A. Salandrino and N. Engheta, Epsilon-near-zero metamaterials and electromagnetic sources: Tailoring the radiation phase pattern, Phys. Rev. B 75 (2007) 155410, 13 pp.

[12] S. Flügge, Practical Quantum Mechanics (Springer, 1994).

[13] S. W. Hawking, Zeta function regularization of path integrals in curved spacetime, Comm. Math. Phys. 55 (1977) 133-148.

[14] E. Elizalde, S. D. Odintsov, A. Romeo, A. A. Bytsenko and S. Zerbini, Zeta Regularization Techniques with Applications (World Scientific, 1994).

[15] K. Kirsten, Spectral Functions in Mathematics and Physics (Chapman and Hall/ CRC, 2001). 
[16] S. K. Blau, M. Visser and A. Wipf, Zeta functions and the Casimir energy, Nucl. Phys. B 310 (1988) 163-180.

[17] U. Leonhardt, Essential Quantum Optics: From Quantum Measurements to Black Holes (Cambridge University Press, 2010).

[18] K. A. Milton, The Casimir Effect: Physical Manifestations of Zero-Point Energy (World Scientific, 2001).

[19] B. S. Kay, Casimir effect in quantum field theory, Phys. Rev. D 20 (1979) 3052-3062.

[20] G. H. Hardy, Divergent Series (AMS Bookstore, 1991).

[21] G. Barton, On the finite difference between divergent sum and integral, J. Phys. A: Math. Gen. 14 (1981) 1009-1027.

[22] S. Goto, R. W. Tucker and T. J. Walton, The electrodynamics of inhomogeneous rotating media and the Abraham and Minkowski tensors I: General theory, Proc. $R$. Soc. Lond. Ser. A Math. Phys. Eng. Sci. 467 (2011) 59-78.

[23] S. Goto, R. W. Tucker and T. J. Walton, The electrodynamics of inhomogeneous rotating media and the Abraham and Minkowski tensors II: Applications, Proc. $R$. Soc. Lond. Ser. A Math. Phys. Eng. Sci. 467 (2011) 79-98.

[24] P. W. Milonni, The Quantum Vacuum: An Introduction to Quantum Electrodynamics (Academic Press San Diego, 1994).

[25] F. S. S. Rosa, D. A. R. Dalvit and P. W. Milonni, Electromagnetic energy, absorption, and Casimir forces. II. Inhomogeneous dielectric media, Phys. Rev. A 84 (2011) 053813 .

[26] E. T. Whittaker and G. N. Watson, A Course of Modern Analysis (Cambridge University Press, 1996).

[27] M. Abramowitz and I. A. Stegun, Handbook of Mathematical Functions: With Formulae, Graphs, and Mathematical Tables (Dover Publications, 1964).

[28] E. Elizalde, Zeta-function regularization is uniquely defined and well, J. Phys. A Math. Gen. 27 (1994) L299-L304.

[29] S. Goto, A. C. Hale, R. W. Tucker and T. J. Walton, Numerical regularization of electromagnetic quantum fluctuations in inhomogeneous dielectric media, Phys. Rev. A 85 (2012) 034103, 4 pp.

[30] H. P. Baltes and E. R. Hilf, Spectra of Finite Systems (BI-Wissenschaftsverlag Mannheim, 1976). 